\title{
Relationship between Solar Energetic Particles and Properties of Flares and CMEs: Statistical Analysis of Solar Cycle 23 Events
}

\author{
M. Dierckxsens ${ }^{1}$ - K. Tziotziou ${ }^{2}$. S. Dalla ${ }^{3}$. \\ I. Patsou $^{2}$ - M. S. Marsh ${ }^{3}$. N. B. Crosby ${ }^{1}$. \\ O. Malandraki ${ }^{2}$. G. Tsiropoula ${ }^{2}$
}

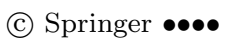

\begin{abstract}
A statistical analysis of the relationship between solar energetic particles (SEPs) and properties of solar flares and coronal mass ejections (CMEs) is presented. SEP events during Solar Cycle 23 are selected which are associated with solar flares originating on the visible hemisphere of the Sun and at least of magnitude M1. Taking into account all flares and CMEs that occurred during this period, the probability for the occurrence of a SEP event near Earth is determined. A strong rise of this probability is observed for increasing flare intensities, more western locations, larger CME speeds and halo CMEs. The correlations between the proton peak flux and these solar parameters are derived for a low $(>10 \mathrm{MeV})$ and high $(>60 \mathrm{MeV})$ energy range excluding any flux enhancement due to the passage of fast interplanetary shocks. The obtained correlation coefficients are: $0.55 \pm 0.07(0.63 \pm 0.06)$ with flare intensity and $0.56 \pm 0.08(0.40 \pm 0.09)$ with the CME speed for $E>10 \mathrm{MeV}(E>60 \mathrm{MeV})$. For both energy ranges, the correlations with flare longitude and CME width are very small or non-existent. Furthermore, the occurrence probabilities, correlation coefficients and mean peak fluxes are derived in multi-dimensional bins combining the aforementioned solar parameters. The correlation coefficients are also determined in different proton energy channels ranging from 5 to $200 \mathrm{MeV}$. The results show that the correlation between the proton peak flux and the CME speed decreases with energy, while the correlation with the flare intensity shows the opposite behavior. Furthermore, the correlation with the CME speed is stronger than the correlation with the flare intensity below $15 \mathrm{MeV}$ and becomes weaker above $20 \mathrm{MeV}$. When the enhancements in the flux profiles due to interplanetary shocks are not excluded, only a small but not very significant
\end{abstract}

\footnotetext{
1 Belgian Institute for Space Aeronomy (BIRA-IASB), Ringlaan 3, 1180 Brussels, Belgium; email:

Mark.Dierckxsens@aeronomie.be

2 IAASARS, National Observatory of Athens, GR-15236

Penteli, Greece

3 Jeremiah Horrocks Institute, University of Central

Lancashire, Preston, PR1 2HE, UK
} 
change is observed in the correlation coefficients between the proton peak flux below $7 \mathrm{MeV}$ and the CME speed.

Keywords: solar energetic particles, solar flares, coronal mass ejections

\section{Introduction}

Solar energetic particles (SEPs) are accelerated at the Sun or in the interplanetary medium during flares and coronal mass ejections (CMEs) reaching energies up to several GeV. SEP events consist of electrons, protons and ions (up to $\mathrm{Fe}$ ) that propagate along the interplanetary magnetic field causing sudden and often long lasting particle radiation enhancements. SEP events show significant variability in their relative abundance and intensity profile, with the maximum of the proton flux near Earth reached in timescales ranging from less than an hour to longer than a day. The characteristics of SEPs are obtained by in situ singular or multiple spacecraft observations. Some SEP profiles exhibit a short period of flux increase varying from a few minutes to half a day when a fast interplanetary shock passes the observation point, called an energetic storm particle (ESP) event, indicating in situ acceleration of particles by the shock (Cane, 1995).

Statistical studies of the properties of SEP events have been carried out for decades (starting with Van Hollebeke, Ma Sung, and McDonald (1975)), with the purpose of broadening our understanding of particle acceleration and propagation. One of the outcomes of such studies has been the suggestion, in the 1990s, of the so-called two-class paradigm for SEPs (Cane, McGuire, and von Rosenvinge, 1986; Reames, 1988, 1999, 2013). The first class of events is associated with impulsive acceleration and generally characterized by a short rise time of the flux time profiles, observation point magnetically well connected with the location of the associated flare, no clear association with a CME or interplanetary shock, high electron/proton and ${ }^{3} \mathrm{He} /{ }^{4} \mathrm{He}$ ratios and enhanced heavier ion content. The second class is referred to as gradual events and predominantly described by a slow rising particle flux, an association with a long lasting flare and CME originating anywhere on the Sun or interplanetary shock, and a relative composition similar to that of coronal material. Later, Cane, Richardson, and von Rosenvinge (2010) studied 280 solar proton events and their associated flares and CMEs, and found a continuum of event properties as opposed to the two classes of the standard SEP paradigm.

In recent years, statistical studies have also become important for space weather forecasting by identifying which types of solar events are most efficient at producing SEPs. Laurenza et al. (2009) carried out an analysis of data from the Geostationary Operational Environmental Satellite (GOES) to develop a technique for short-term warning of SEP events using flare properties and type III radio emissions. Several other authors have studied the dependence of SEP characteristics on various flare parameters (Kurt et al., 2004; Belov et al., 2005; Park et al., 2010; Cliver et al., 2012), CME characteristics (Kahler, 2001; Gopalswamy et al., 2008; Park, Moon, and Gopalswamy, 2012) or both (Wang, 2006; 
Cane, Richardson, and von Rosenvinge, 2010; Hwang et al., 2010; Miteva et al., 2013; Richardson et al., 2014). Some of these studies also included information on type II (Wang, 2006; Gopalswamy et al., 2008; Richardson et al., 2014) and type III (Cane, Richardson, and von Rosenvinge, 2010; Richardson et al., 2014) radio bursts.

This article presents a statistical analysis of SEP events and their associated flares and CMEs during Solar Cycle 23. The purpose of this study is twofold: 1) to characterize the probabilities of SEP occurrence based on a number of characteristics of flares and CMEs and 2) for the solar events that did result in observed SEPs, to identify quantitatively the relationship between the associated flare and CME properties and the peak value of the proton flux during the SEP event. This analysis was performed as part of the EU FP7 project COMESEP (COronal Mass Ejections and Solar Energetic Particles; Crosby et al., 2012; http://www.comesep.eu). The main objectives of this project were the development of tools to forecast geomagnetic and SEP radiation storms, and the integration of these tools into an operational, fully automated space weather alert system. The results of this statistical analysis are incorporated into the SEP forecast tools of COMESEP.

Flare intensity and location as well as CME speed and angular width are known to influence the occurrence and characteristics of SEP events, as demonstrated in the aforementioned studies. As far as the location of the flare is concerned, the dependence on flare longitude has been known since the earliest studies (e.g. Van Hollebeke, Ma Sung, and McDonald (1975); Cane, Reames, and von Rosenvinge (1988)), while any influence of latitude for near-Earth SEP events is unclear (Dalla and Agueda, 2010). Radio data is not considered in our analysis as there are currently no near real-time radio burst detection tools available that can be used for forecast purposes. While most studies have analyzed SEP events for protons within a specific energy range, typically around 10 or $20 \mathrm{MeV}$, here we also consider a higher energy range dominated by protons of about $60 \mathrm{MeV}$ (i.e. the range $E>60 \mathrm{MeV}$ ), in addition to a low energy range $(E>10 \mathrm{MeV})$. These two energy ranges are chosen to ensure adequate coverage of different types of technological and biological effects induced by SEP radiation. Hence, we investigate the dependence of the SEP peak flux in these two energy ranges and the occurrence probability on flare intensity and longitude, and CME speed and width. In this work, we go a step further by examining how the quantities under study vary when combining flare and CME parameters, by exploring the energy dependence of the correlation coefficients between the peak flux and these solar parameters, and by assessing how not excluding the flux originating from an ESP influences these values.

The event lists and SEP data used for this analysis are described in Section 2 and our methodology is explained in detail in Section 3 Section 4 presents our results which are discussed and compared with previous works in Section 5 ,

\section{Event Lists and Associated Data}

Our statistical studies cover the years 1997-2006, i.e. mostly Solar Cycle 23, to take advantage of the Large Angle and Spectrometric Coronagraph (LASCO) 
on-board the Solar and Heliospheric Observatory satellite (SOHO). The SEP dataset and the comprehensive lists of events (SEPs, solar flares and associated CMEs) used for this analysis are described in the following sections.

\subsection{SEP Data}

For a statistical study of the characteristics of SEP events, it is essential to use a well verified dataset. Therefore, we use the Solar Energetic Particle Environment Modelling (SEPEM) reference proton dataset (Crosby et al. (2014); http://dev.sepem.oma.be) from the European Space Agency (ESA). Within this project, data from the Space Environment Monitor (SEM) on GOES and the Goddard Medium Energy (GME) instrument on the Interplanetary Monitoring Platform (IMP-8) for the period 1973 to 2013 were cleaned, rebinned, crosscalibrated and merged to provide a uniform dataset of ten differential energy channels exponentially distributed in the range from 5 to $200 \mathrm{MeV}$. The SEP data used in our analysis constitute, thus, a more consistent dataset than those used in previous studies that relied on data obtained by single or multiple satellites and instruments.

\subsection{SEP Event Lists}

Our study of SEP occurrence probabilities and peak fluxes relies on two SEP event lists as will be described in Section 4. In both lists, only events associated with solar flares of at least magnitude M1 and with an identified source longitude within the visible earthward solar hemisphere $\left[-90^{\circ}, 90^{\circ}\right]$ were retained.

CRR2010 List The first SEP list is a subset of the list of Cane, Richardson, and von Rosenvinge (2010) (hereinafter referred to as CRR2010) which they compiled from the analysis of $>25 \mathrm{MeV}$ proton data from the IMP8/GME and SOHO/ERNE (Energetic and Relativistic Nuclei and Electron) instruments. The information on the solar flare and CME associated with each event was also obtained from CRR2010 and verified with the original sources (see Section 2.3). The values for the angular width were re-evaluated by CRR2010 for asymmetric halo CMEs and in some cases differ substantially from those provided in the SOHO/LASCO CME catalogue. After applying the aforementioned criteria to the associated flare, the subset contains 160 events which will be referred to in the following as the CRR2010 list.

SSE List The second SEP event list is a subset of the reference proton event list derived within the SEPEM project based on the proton energy range $7.23-$ 10.45 MeV (Jiggens et al., 2012). The majority of events in this list have a clear counterpart in the CRR2010 list. In some cases SEPEM events are in fact the sum of several physical SEP events due to the requirement of a minimum dwell time of 24 hours between consecutive events. These SEPEM events were split up into separate events based on the start times of events in CRR2010. An example of a SEPEM event containing more than one CRR2010 event can be seen in Figure 1. The information of the associated flare and CME was obtained from 


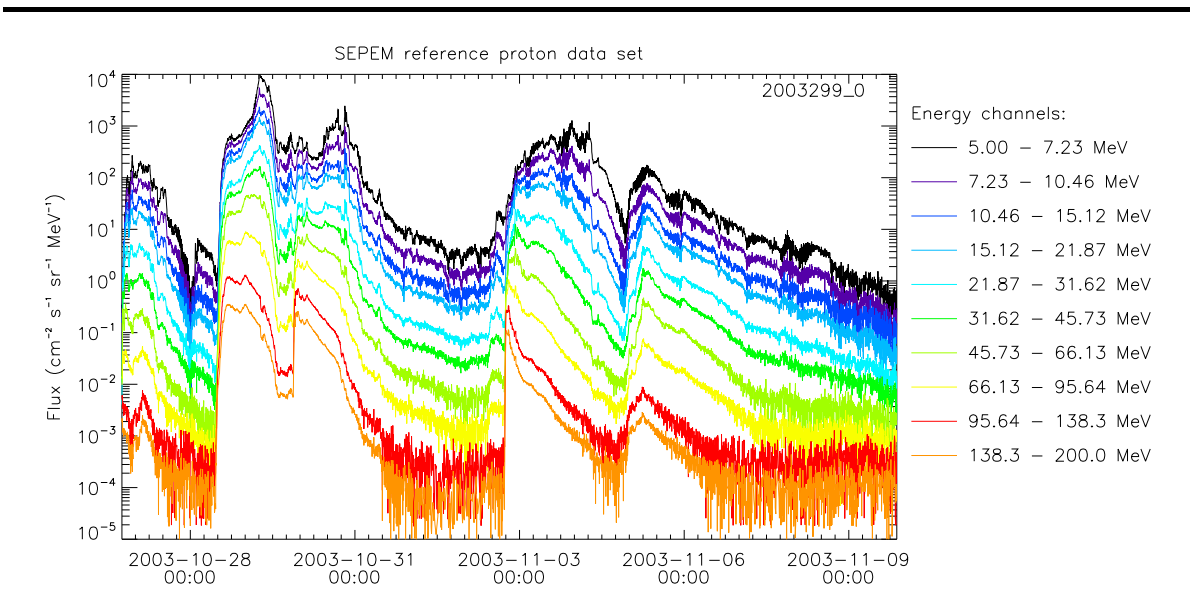

Figure 1. The flux-time profile in the SEPEM reference proton data channels of the event starting on October 26, 2003. Separate enhancements during the event duration can be observed corresponding to seven events identified by CRR2010.

the CRR2010 list. For the few events without a clear counterpart in this list, we associated solar activity using the sources mentioned in Section 2.3. This resulted in a total of 90 events satisfying the flare criteria and will be referred to in the following as the SEPEM Sub Event (SSE) list. The full list of SSE events, including the parameters of the associated flares and CMEs, can be found in the Appendix in Table 4

The difference between the number of SEP events in the two lists, 160 and 90 SEPs respectively, stems from both the different energy ranges used for the compilation of the two lists, resulting from different satellites/instruments, and the different threshold criteria used for classifying a flux enhancement as a SEP event.

\subsection{Solar Eruptive Event Lists}

In order to study the SEP occurrence probability, several solar eruptive event lists (flares and CMEs) were collected. These lists and their original sources were also used to verify and complement the associations with the SEP events as described in Section 2.2 ,

Flare List A subset of the NOAA GOES X-ray solar flare catalogue (available at http://www.ngdc.noaa.gov/stp/spaceweather.html) was obtained by applying the same time range, flare intensity and location criteria as for the SEP lists. This resulted in a sample of 1298 flares.

Flare-CME List Events from the SOHO/LASCO CME Catalogue (available at http://cdaw.gsfc.nasa.gov/CME_list/) were associated by Dumbovic et al. (2014) with solar flares from the NOAA X-ray solar flare catalogue using both temporal and spatial criteria. All flares within one hour of each CME liftoff 
were selected. For non-halo CMEs and flares with source location information, the difference between the CME and flare position angles was required to be less than half the CME angular width. In cases where multiple flares satisfy the above criteria, only the strongest flare was chosen as the CME-associated flare. Applying the same criteria as for the flare list above, a total of 438 flare-CME pairs were obtained for our analysis.

\section{Methodology}

This section describes the methodology used for the derivation of SEP occurrence probabilities in solar eruptive events, the identification of the ESP events, the fitting to the energy spectra of the proton peak flux during the SEP event, and the derivation of integral fluxes.

\subsection{Probabilities of SEP Occurrence in Solar Eruptive Events}

Given a subset $j$ of flare or CME events characterized by certain parameters (e.g. flare intensity or CME speed in a specified range), the probability of SEP occurrence $P_{j}$ for subset $j$ is defined as:

$$
P_{j}=\frac{N_{j}^{S E P}}{N_{j}}
$$

where $N_{j}$ is the total number of solar eruptive events in the subset $j$ and $N_{j}^{S E P}$ the number of those events that resulted in an observed SEP event.

The uncertainty associated with the probability $P_{j}$ is derived according to the binomial proportion confidence interval as $\pm \sqrt{P_{j}\left(1-P_{j}\right) / N_{j}}$. This corresponds to a $68 \%$ confidence level when the sample is described by a normal distribution, which is however a weak assumption for the bins containing a small number of events.

\subsection{Identification of ESP Events}

An ESP is an enhancement in particle fluxes caused by a local interplanetary shock wave. In the interplanetary medium, shocks accelerate lower energy particles more efficiently, making ESP enhancements usually much more apparent in the lower energy channels (see e.g. Channok et al. (2005)). For each event in our SSE list, the time profiles of the proton flux in all energy channels were examined for ESP-like increases during the decay phase of the initial event. Lists of interplanetary shocks observed at the Advanced Composition Explorer (ACE) and Wind spacecraft (http://www.cfa.harvard.edu/shocks) were used to verify whether a shock was observed at $1 \mathrm{AU}$ around the time of these ESP-like enhancements. An ESP could be identified during 45 out of the 90 events in our SSE list. The ESP onset is determined as the time when the proton flux starts to rise again after the initial decay. 

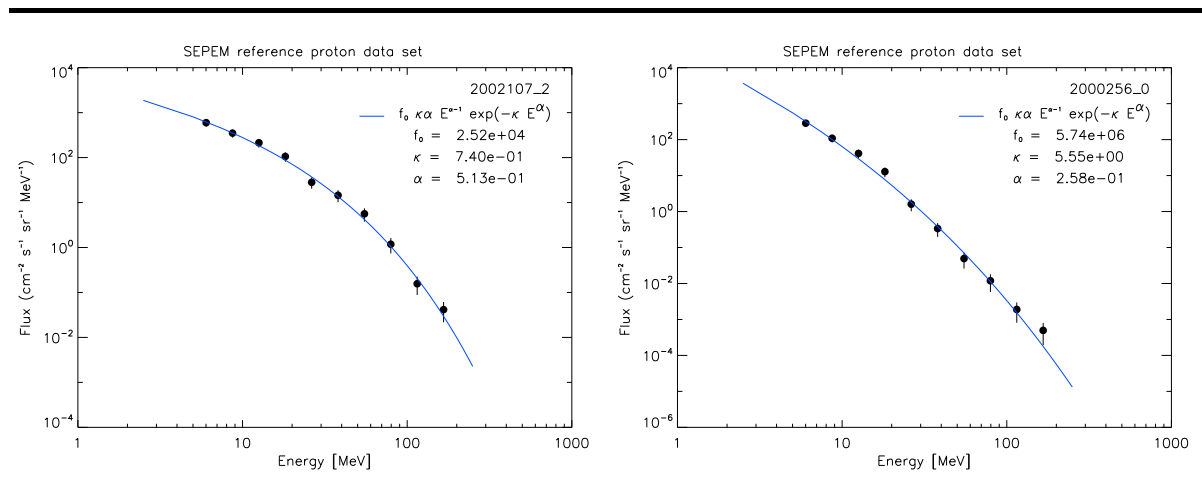

Figure 2. The energy spectrum of the proton peak flux for the event on 21 April 2002 (left) and on 12 September 2000 (right) showing two different but typical shapes. The black dots show the differential flux measured in each energy channel, while the blue line represents the fit with a Weibull function. The resulting fit parameters are also shown.

\subsection{Proton Peak Fluxes and Energy Spectrum Fit}

To obtain the peak flux in the ten differential SEPEM energy channels, the maximum observed flux is determined before any identified ESP onset time during the event (see Section 3.2). The energy in each channel is determined as the square root of the product of the lower and upper limits of the corresponding channel.

The obtained spectrum for each event is fitted with a Weibull function, which was shown to describe SEP spectra accurately up to GeV energies (Xapsos et al., 2000) and has the form:

$$
\frac{d \phi}{d E}=f_{0} \kappa \alpha E^{\alpha-1} e^{-\kappa E^{\alpha}}
$$

with $\phi$ representing the particle flux, $E$ the energy of the particle measured in $\mathrm{MeV} /$ nucleon, $f_{0}$ a normalization factor, and $\kappa$ and $\alpha$ two parameters that determine the shape of the distribution. An example of this fit for two different spectral shapes can be seen in Figure 2

An uncertainty on the peak flux values was required in order for the fits to properly converge. Unfortunately, these values are not provided in the SEPEM data. We have chosen to let the relative uncertainty on the flux value increase with the square root of the energy to reflect the increase in the uncertainty with the decrease in flux (particle count). Since the resulting uncertainties on the fit parameters are not used in the subsequent analysis, an arbitrary uncertainty of $10 \%$ was assigned to the SEPEM reference energy channel $(7.23-10.45 \mathrm{MeV})$. Various different dependencies on the flux and energy were explored, but it was found that this only had a very small effect on the derived integral peak fluxes that are described in Section 3.4 .

\subsection{Determination of Integral Peak Fluxes}

Within a forecasting framework, it is useful to provide forecasts of peak fluxes for a set of standard integral energy ranges. Here we have chosen to derive 
the dependence of the impact on the solar parameters for the energy ranges $E>10 \mathrm{MeV}$ and $E>60 \mathrm{MeV}$. To construct an integral peak flux from the SEPEM fluxes, the results from the fit to the peak flux spectrum as described in Section 3.3 are used. By integrating Equation 2 and filling in the fit parameters, the integral peak fluxes in a desired energy range can be easily derived. The resulting values of the integral peak flux in the aforementioned energy ranges for each event in the SSE list can be found in Table 4 in the Appendix.

\section{Results}

This section presents our results on the probabilities of SEP occurrence in solar eruptive events and the SEP event characteristics based on the methodologies described in Section 3. We remind that the results described in Section 4.1 were derived using the larger CRR2010 list, since in this analysis we are mainly interested in SEP occurrence probabilities as a function of flare and CME characteristics, irrespective of the particular SEP profile characteristics. The results described in Section 4.2 were derived using the SSE list since it contains the detailed SEP profile characteristics based on the cleaned dataset.

\subsection{Probabilities of SEP Occurrence}

We have derived the probabilities of SEP occurrence (see section 3.1) by determining the fraction of flares in the Flare or Flare-CME list resulting in an entry in the CRR2010 list as a function of several solar event parameters. Since the COMESEP SEP forecast tools are only triggered following the observation of a soft X-ray solar flare, the combinations chosen in the following sections are based on the potential availability of data after the measurement of the flare magnitude. For comparison, the same quantities have been derived as a function of the flare parameters for the SSE list used to derive the proton peak fluxes.

\subsubsection{Probabilities as a Function of Flare Parameters}

Figure 3 shows the distributions of flare magnitude and longitude of the samples containing the 1298 flares and 160 SEP-associated flares from the CRR2010 list used for the derivation of SEP occurrence probabilities. The distributions for the 90 SEP-associated flares of the SSE list are also shown. In the CRR2010 list, 104 SEP events are related to M-class flares and 56 events to X-class flares, while the respective numbers in the SSE list are 50 and 40 . Within a solar cycle, the frequency of flares drops considerably with increasing magnitude while there is no preference for the longitude location on the solar disk, as our distributions clearly demonstrate. As for flares resulting in an observed SEP event, the decline in the number of events as a function of flare magnitude is less steep, while there is a clear westward preference in location.

Probabilities are derived as a function of flare magnitude only, and as a function of both flare magnitude and longitude. Given the relatively small number of SEP events (160) compared to the total flare sample (1298), the analysis is 

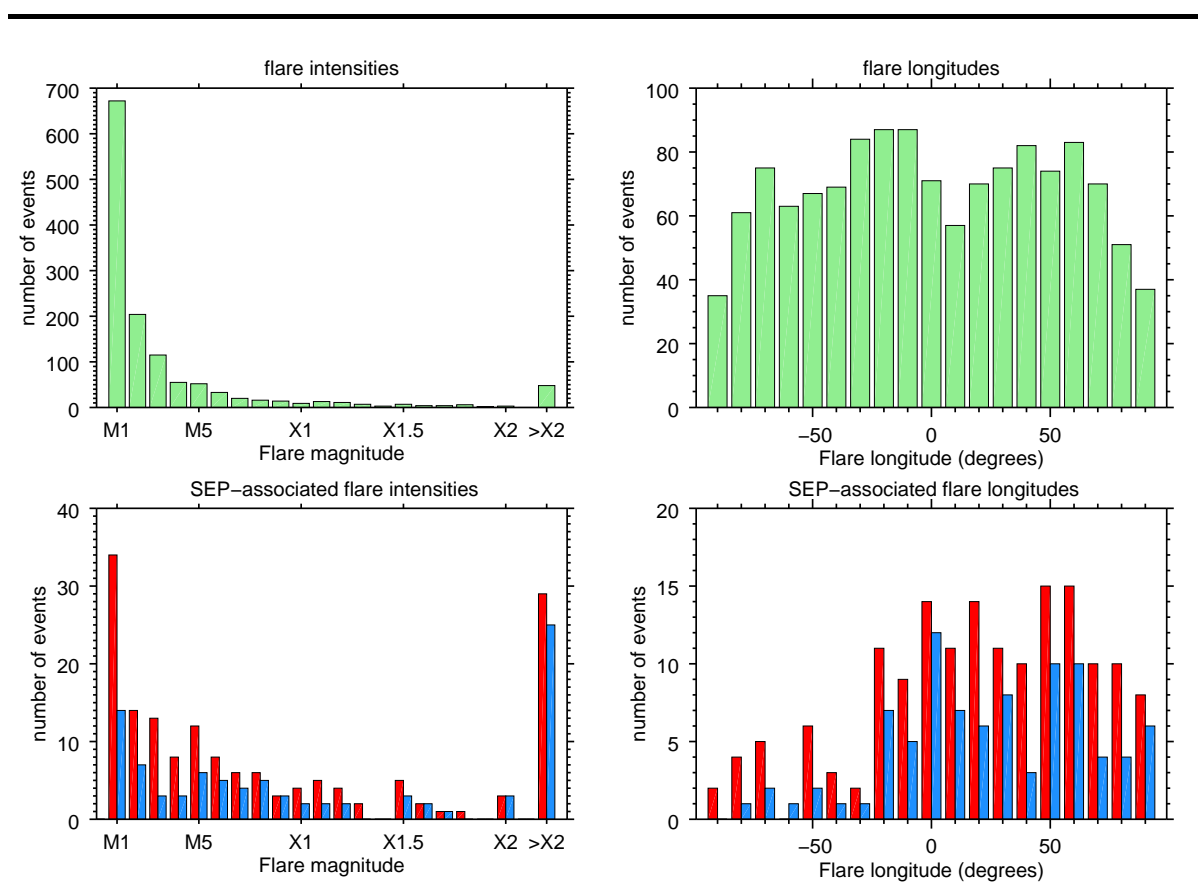

Figure 3. Top row: distributions of flare magnitude (left) and flare longitude (right) of the sample of 1298 flares used for the analysis of Section 4.1.1 Bottom row: distributions of the respective parameters of the flares associated to the events in the CRR2010 list (red bars) and in the SSE list (blue bars).

performed within a limited number of flare magnitude and flare longitude bins: five flare magnitude bins, namely [M1-M3.9], [M4-M6.9], [M7-M9.9], [X1-X4.9] and $[\geq \mathrm{X} 5]$, and five longitude bins, namely $\left[-90^{\circ},-71^{\circ}\right],\left[-70^{\circ},-31^{\circ}\right],\left[-30^{\circ}, 29^{\circ}\right]$, $\left[30^{\circ}, 69^{\circ}\right]$ and $\left[70^{\circ}, 90^{\circ}\right]$. The bin widths were defined by taking into account the distributions presented in Figure 3 . They are equidistant for longitude, as the distribution is rather flat, while for flare magnitude the choice was based both on the presence of sufficient number of events per bin and the importance of specific flare magnitudes for SEP occurrence. It should be noted that a different choice of binning resulted in qualitatively similar results as presented here. Figure 4 (and Table 5in the Appendix) exhibit a clear rise in SEP occurrence probabilities for increasing flare magnitude. The same probabilities derived for the SSE list are also shown. Although probabilities are smaller compared to the ones obtained with the CRR2010 list as a result of the lower number of selected SEP events, their flare magnitude dependence shows a rather good qualitative agreement.

Figure 5 (and Table6 in the Appendix) show that the derived SEP occurrence probabilities increase for both stronger and more westward flares. The probabilities derived with the SSE list exhibit a very similar behavior to the values derived with the CRR2010 list although they are generally somewhat lower. Some of the most significant decreases in the SSE SEP probabilities compared to the respective CRR2010 SEP probabilities are present in the $[\mathrm{X} 1, \mathrm{X} 4.9]$ flare magnitude bin for both east $\left[-90^{\circ},-71^{\circ}\right]$ and west $\left[70^{\circ}, 90^{\circ}\right]$ longitude bins. A detailed investigation of the events not present in the SSE list that fall in these 


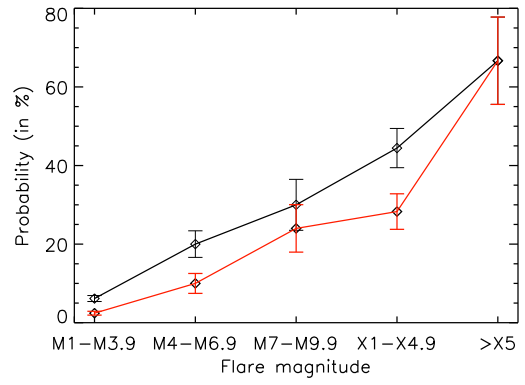

Figure 4. SEP occurrence probabilities (in \%) and their respective errors as a function of flare magnitude derived from the CRR2010 list (black line) and the SSE list (red line) for five different flare magnitude bins: [M1,M3.9], [M4,M6.9], [M7,M9.9], [X1,X4.9] and [ $\geq \mathrm{X} 5]$.
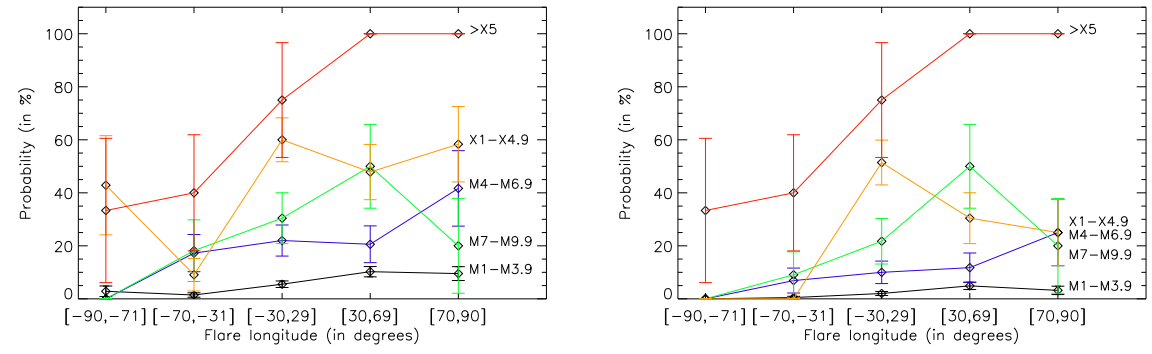

Figure 5. SEP occurrence probabilities (in \%) and their respective errors as a function of flare magnitude and location derived from the CRR2010 list (left) and the SSE list (right) for five longitude bins: $\left[-90^{\circ},-71^{\circ}\right],\left[-70^{\circ},-31^{\circ}\right],\left[-30^{\circ}, 29^{\circ}\right],\left[30^{\circ}, 69^{\circ}\right]$ and $\left[70^{\circ}, 90^{\circ}\right]$, and five flare magnitude bins: $[\mathrm{M} 1, \mathrm{M} 3.9]$ (black), [M4,M6.9] (blue), [M7,M9.9] (green), [X1,X4.9] (orange) and $[\geq \mathrm{X} 5]$ (red).

bins has shown that they are mainly electron dominated events, which are not included due to the different selection criteria.

\subsubsection{Probabilities as a Function of Flare and CME Characteristics}

Figure 6 shows the distributions of flare magnitude, flare longitude, CME velocity and CME width for the 438 flare-CME sample and the 118 SEP-associated events used to derive the SEP probabilities as a function of flare and CME characteristics. Out of these 118 SEP events, 78 are associated with M-class flares while 40 are associated with X-class flares. The flare magnitude and longitude distributions show similarities with the respective distributions of the sample of 1298 flares discussed in the previous section (see Figure 3, top row), suggesting that this CME-associated flare sample is quite representative of the initial flare sample. The majority of CME velocities are in the range from 0 to $1500 \mathrm{~km} \mathrm{~s}^{-1}$, with a tail in the distribution extending to almost $3000 \mathrm{~km} \mathrm{~s}^{-1}$. In the CME width distribution, there seem to be two distinct and similarly sized populations: one of CMEs with a width in the range $\left[0^{\circ}, 300^{\circ}\right]$ and one of halo CMEs $\left(360^{\circ}\right)$. When only the subset resulting in SEP events is considered (118 events) we 

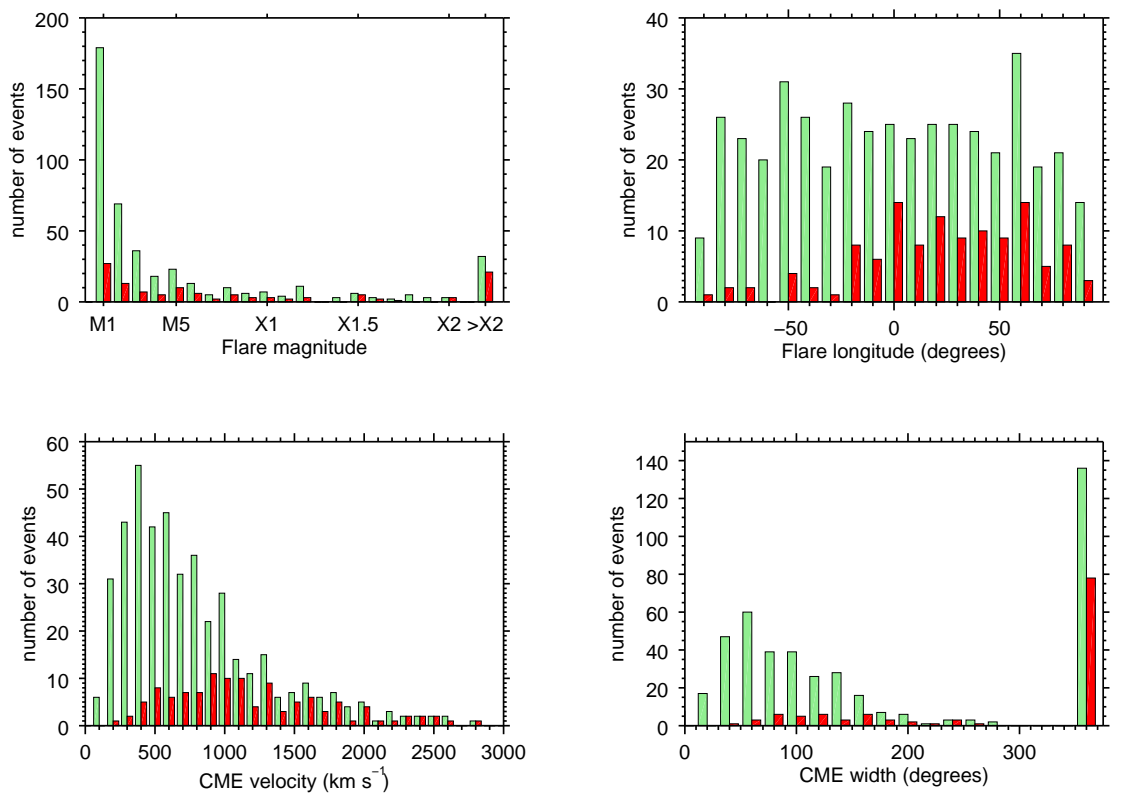

Figure 6. Distributions of flare magnitude, flare longitude, CME velocity and CME width for the 438 CME-associated flare sample (green bars) and the 118 SEP-associated events (red bars). In the CME width distribution the $360^{\circ}$ bin represents halo CMEs.

notice again a clear preference for westward longitudes and a much higher mean CME velocity of the order of $1000 \mathrm{~km} \mathrm{~s}^{-1}$.

Given the small number of both SEPs (118) and CME-associated flares (438), the derivation of SEP occurrence probabilities has been performed for a small number of flare magnitude, flare longitude, and CME velocity bins to limit statistical fluctuations: three flare magnitude bins, namely [M1-M3.9], [M4-M9.9] and $[\geq \mathrm{X} 1]$; two longitude bins, namely $\left[-90^{\circ},-1^{\circ}\right]$ and $\left[0^{\circ}, 90^{\circ}\right]$; and three $\mathrm{CME}$ velocity bins, namely $[0,499],[500,999]$ and $[\geq 1000] \mathrm{km} \mathrm{s}^{-1}$. Again the choice of binning is based on the distributions of Figure 6 and the requirement to have sufficient number of events per bin. As for the dependence on CME width, two distinct cases are considered: non-halo $\left(<360^{\circ}\right)$ and halo $\left(360^{\circ}\right)$ CMEs. As Figure 7 clearly demonstrates, the ratio between the distribution of the SEP-associated sample and the full sample is rather constant for halo CMEs, except for the very fast CMEs $\left(\geq 2000 \mathrm{~km} \mathrm{~s}^{-1}\right)$. For the very fast halo CMEs the sample numbers are, however, very small. Since there is no particularly strong dependence on the CME velocity for halo CMEs, the SEP occurrence probabilities will only be derived as a function of flare characteristics. This is in contrast to the non-halo CMEs which clearly show a strong dependence on the CME velocity. The difference in the dependency on the CME velocity between the two CME categories is further explored in Section 4.1.3.

CME velocity and width are both derived simultaneously following the detection of a CME in SOHO/LASCO. This is in contrast to the flare parameters 

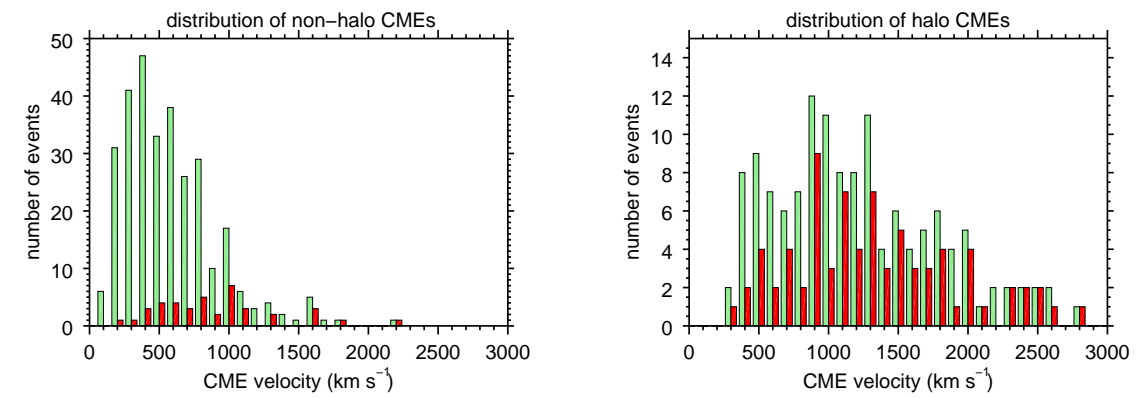

Figure 7. Distribution of non-halo (left panel) and the halo (right panel) CME velocity for the CME-associated flare sample (green bars) and for the corresponding SEP-associated sample (red bars).

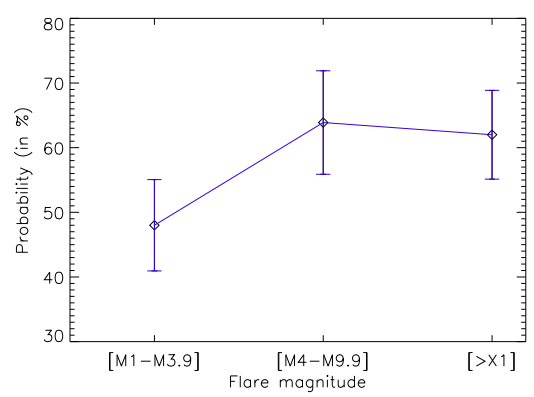

Figure 8. SEP occurrence probabilities (in \%) and their respective errors as a function of flare magnitude for halo CMEs.

since the location is not always known when a flare is observed as they are obtained from different instruments. We therefore consider the following cases of SEP occurrence probabilities: a) as a function of flare magnitude alone for halo CMEs, b) as a function of flare magnitude and CME velocity for non-halo CMEs, c) as a function of both flare magnitude and longitude for halo CMEs, and d) as a function of flare magnitude and longitude and CME velocity for non-halo CMEs.

Figure 8 (and Table 7 in the Appendix) show the derived SEP occurrence probabilities as a function of flare magnitude for halo CMEs while Figure9 (and Table 8 in the Appendix) show these quantities as a function of flare magnitude and CME velocity for non-halo CMEs. For halo CMEs, the SEP occurrence probability increases with flare magnitude but seems to stay almost constant for flares with magnitudes larger than M5. For non-halo CMEs, with the exception of SEPs associated with flares in the [M4-M9.9] magnitude bin and CMEs faster than $1000 \mathrm{~km} \mathrm{~s}^{-1}$, there is a clear monotonic dependence on both flare magnitude and CME velocity.

Figure 10 (and Table 9 in the Appendix) show the derived SEP occurrence probabilities as a function of flare magnitude and longitude for halo CMEs. Probabilities do generally show a monotonic dependence with flare magnitude and are 


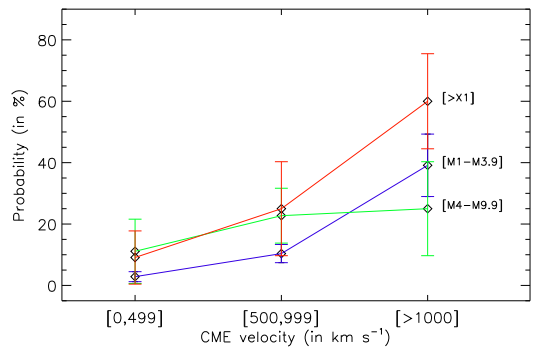

Figure 9. SEP occurrence probabilities (in \%) and their respective errors as a function of flare magnitude and CME velocity for non-halo CMEs.

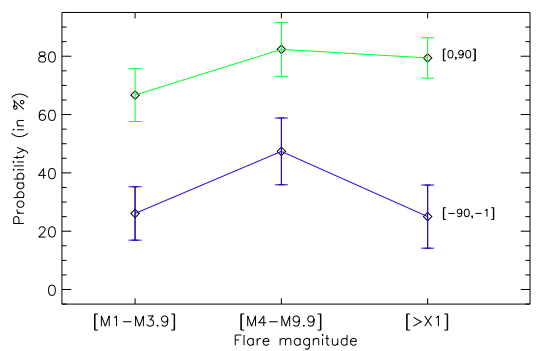

Figure 10. SEP occurrence probabilities (in \%) and their respective errors as a function of flare magnitude and longitude for halo CMEs.

much higher for events originating at the western solar hemisphere as noted before. Figure11 (and Table10 in the Appendix) show the derived SEP occurrence probabilities as a function of flare magnitude, flare longitude and CME velocity for non-halo CMEs. Again, probabilities are generally higher for larger flares and westward flare longitudes, while there also exists a clear monotonic dependence on CME velocity, with fast CMEs resulting in higher SEP probabilities. There is still an exception for the fastest CMEs in the [M4,M9.9] magnitude bin as previously noted, which is now clearly associated with westward flares.

\subsubsection{Probabilities as a Function of CME Characteristics}

All CMEs of the studied sample are associated with flares and hence SEP occurrence probabilities were derived by taking into account both flare and CME characteristics. As a complementary analysis to the aforementioned combinations, the dependency on CME characteristics only was also studied. Figure 12 (and Table 11 in the Appendix) show the derived SEP occurrence probabilities as a function of CME velocity only for the 438 flare-CME sample, regardless of flare magnitude and location, and for the two considered categories defined by CME width: non-halo and halo CMEs. For all cases, the SEP occurrence probability increases with CME velocity, where the behavior is much more pronounced for non-halo CMEs compared to the halo CMEs. For the latter category, the probability is considerable even for slow CMEs while for non-halo CMEs, 


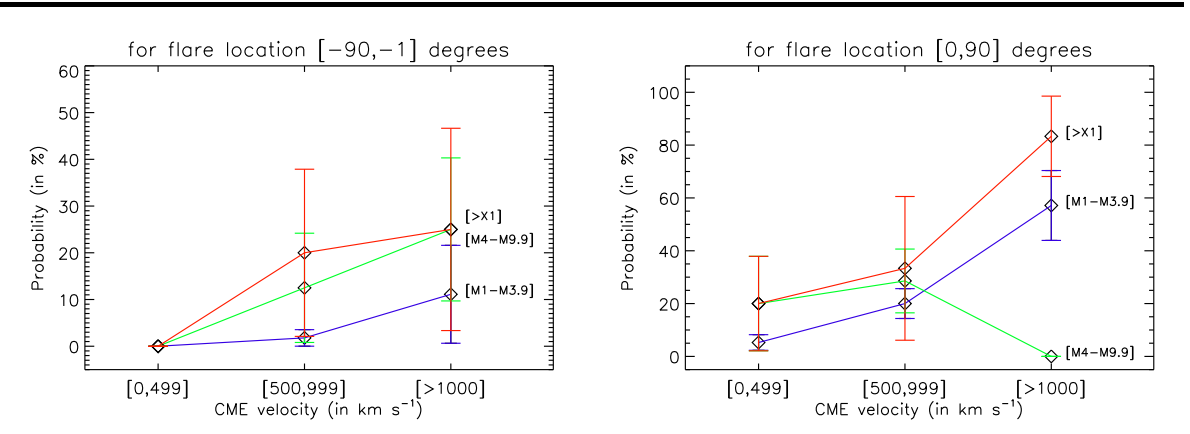

Figure 11. SEP occurrence probabilities (in \%) and their respective errors as a function of flare magnitude, flare longitude and CME velocity for non-halo CMEs.
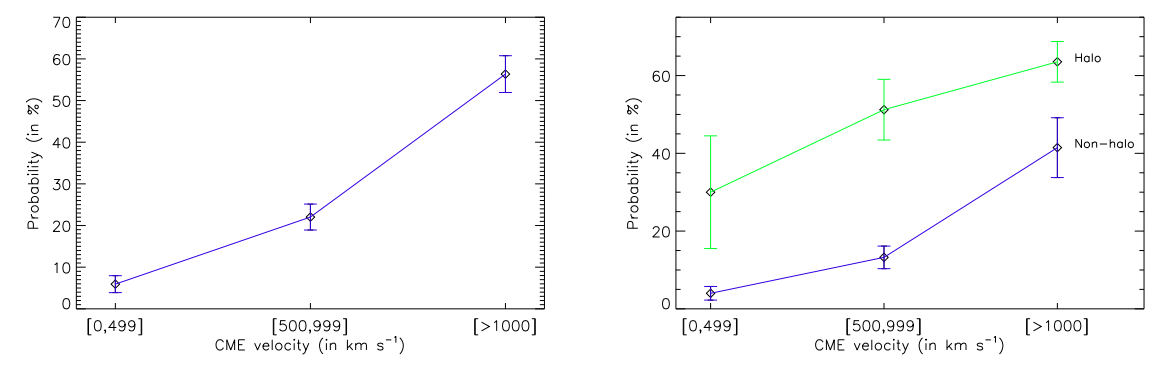

Figure 12. SEP occurrence probabilities (in \%) and their respective errors as a function of CME velocity for all CMEs (left panel) and for non-halo and halo CMEs separately (right panel).

the probability becomes considerable only for the fastest CMEs. Figure 12 also shows that regardless of CME velocity, the probability for a SEP event to occur following a halo CME is significantly larger than after a non-halo CMEs.

\subsection{Proton Peak Flux}

In this section, the relationships between the proton peak fluxes of the SEP events and their associated flares and/or CMEs are derived. The integral peak fluxes in the energy ranges $E>10 \mathrm{MeV}$ and $E>60 \mathrm{MeV}$ as described in Section 3.4 for the events in the SSE list are used. Out of the 90 events in this list, two do not surpass the original flux threshold used to construct the SEPEM reference proton event list and are therefore not considered in this part of the analysis. Their distributions can be seen in Figure 13 and show that the larger events occur less frequently. The sharp rise in the distribution for $E>10 \mathrm{MeV}$ is due to the threshold applied to define a SEP event. The distribution for $E>60 \mathrm{MeV}$ shows a much slower rise which is a consequence of the variety in spectral shapes of SEP events.

The solar eruptive event parameters considered in this analysis are the same as in Section 4.1] flare intensity and longitude, and CME speed and angular width. Out of the 88 events in the SSE list which are related to $\geq$ M1 flares on the visible disk, 78 were associated with a CME. The distributions of the flare 

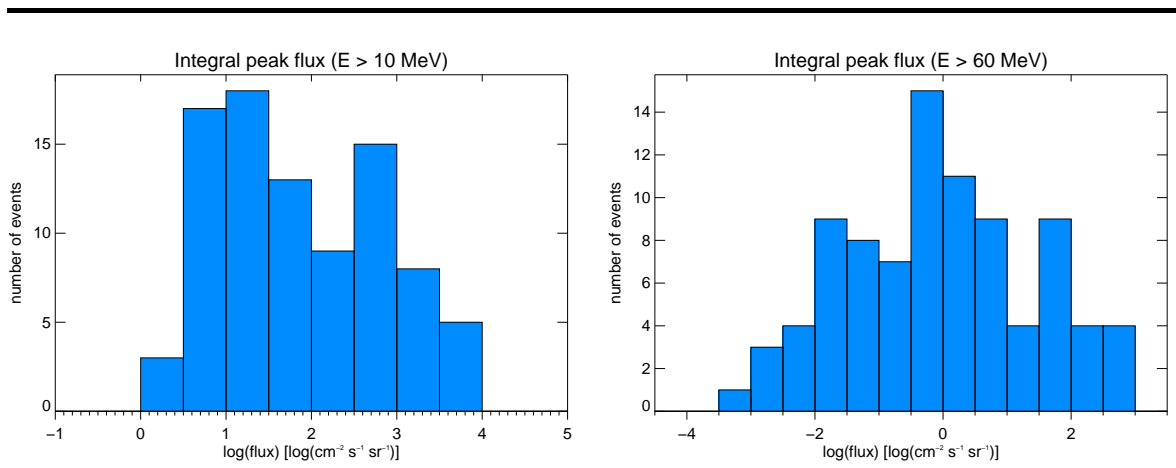

Figure 13. Distributions of SEP integral peak fluxes (in logarithmic scale) for protons with $E>10 \mathrm{MeV}$ (left) and $E>60 \mathrm{MeV}$ (right) for the events in the SSE list.
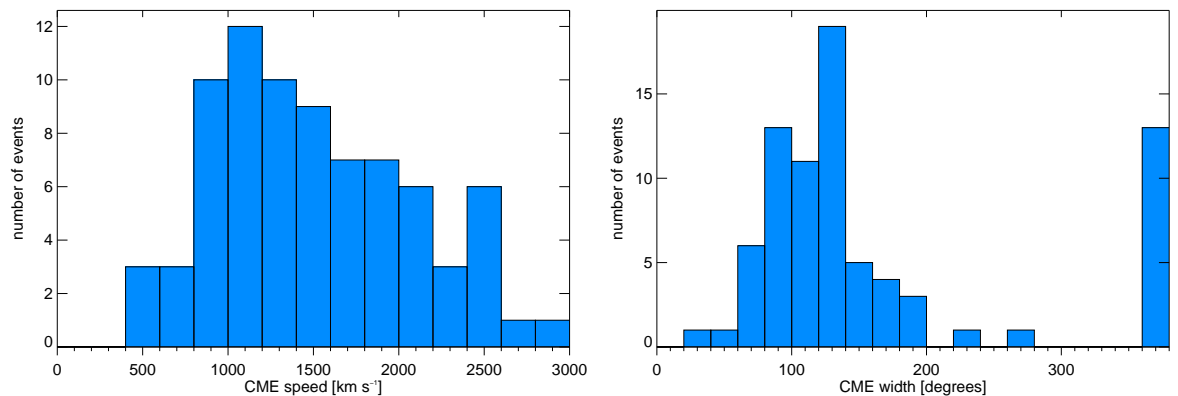

Figure 14. Distributions of the speed (left) and angular width (right) of the CMEs associated with the events in the SSE list.

parameters associated with SEPs are shown in the bottom row of Figure 3, while the CME parameters can be seen in Figure 14. To study the dependence of the peak flux on these solar parameters, the Pearson product-moment correlation coefficient between these two quantities is calculated. The uncertainties on these coefficients are determined using the bootstrap method (Wall and Jenkins, 2003). In total $\mathrm{N}$ events are randomly drawn from a sample of $\mathrm{N}$ events, where the same event can be chosen multiple times. This process is repeated many times, and for each new sample the correlation coefficient is calculated. The average of the resulting distribution is very close to the correlation coefficient of the original sample and the standard deviation provides an estimate of the uncertainty.

\subsubsection{Peak Flux as a Function of Individual Flare and CME Parameters}

Figure 15] shows that a moderate correlation exists between the proton peak flux and flare intensity, and is slightly larger for the higher energy range. A moderate correlation can also be observed in Figure 16 between the proton peak flux and CME speed, which is larger for the lower energy range. The integral peak flux for $E>10 \mathrm{MeV}$ versus the flare longitude and CME angular width is shown in Figure 17, and in both cases, no significant correlation is observed. All derived correlation coefficients for both peak fluxes of $E>10 \mathrm{MeV}$ and $E>60 \mathrm{MeV}$ protons are given in Table 1 . 

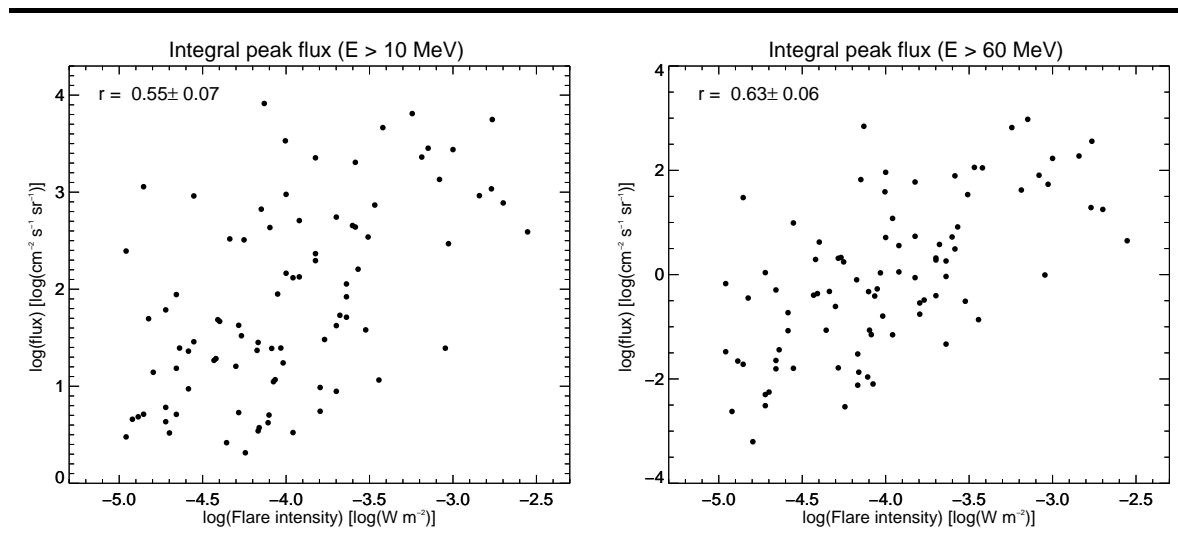

Figure 15. Scatter plots of the logarithm of the proton peak flux as a function of the logarithm of the flare intensity for $E>10 \mathrm{MeV}$ (left) and $E>60 \mathrm{MeV}$ (right). The correlation coefficient $\mathrm{r}$ and its uncertainty is also shown.
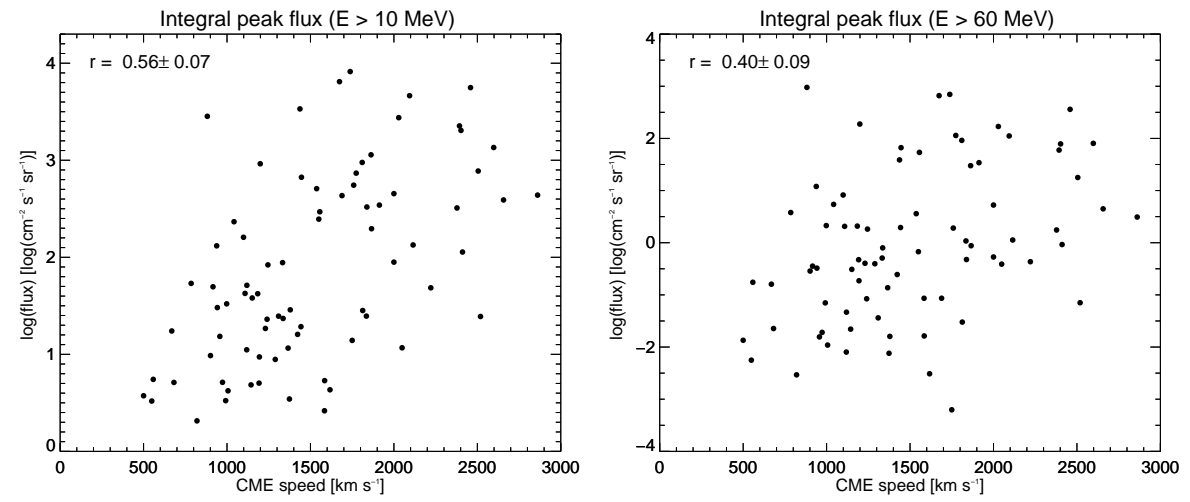

Figure 16. Scatter plots of the logarithm of the proton peak flux as a function of the CME speed for $E>10 \mathrm{MeV}$ (left) and $E>60 \mathrm{MeV}$ (right). The correlation coefficient $\mathrm{r}$ and its uncertainty is also shown.

Since there is a difference between the values of the different energy ranges, a potential energy dependence of these quantities was further investigated. The correlation coefficients between the peak flux and flare intensity and CME speed were derived for each individual SEPEM energy channel and are listed in Table2 and shown in Figure 18, It can be seen that the correlation between the peak flux and flare intensity increases with energy up to $30 \mathrm{MeV}$ and then stays roughly constant. The correlation with the CME speed shows the opposite behavior: the value decreases with energy at least until above $100 \mathrm{MeV}$. It is also interesting to observe that the correlation with CME speed is larger than the correlation with flare intensity for the lowest energies, while they are smaller for the higher energies.

Table 2 also lists the correlation coefficients when the enhancement in flux due to an ESP is not excluded from the peak flux determination. Taking into account that values within the same energy channels are highly correlated, a small but not very significant difference can only be observed for the correlation 

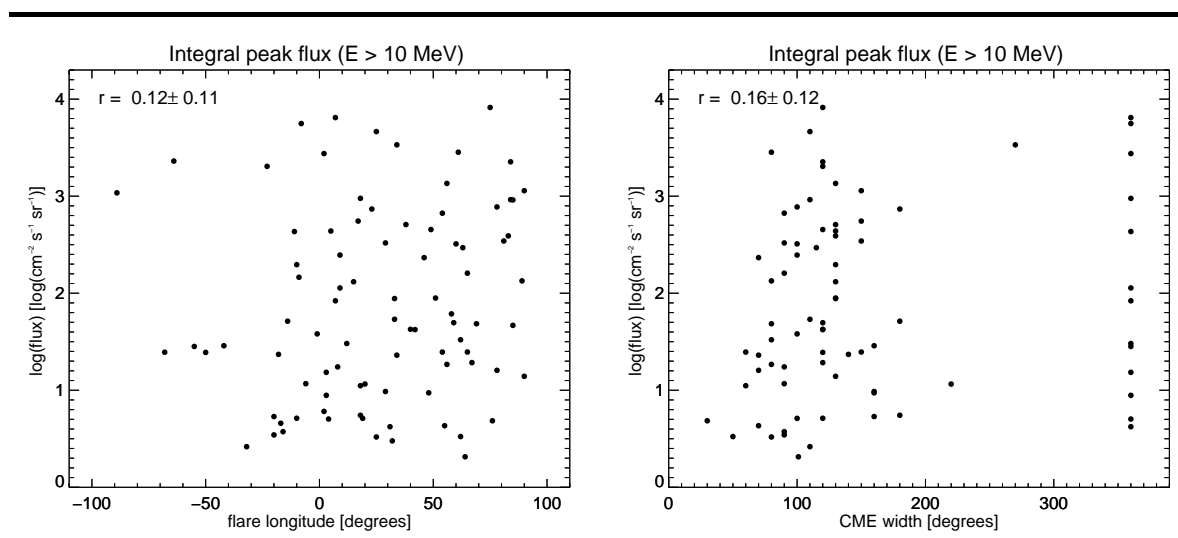

Figure 17. Scatter plots of the logarithm of the proton peak flux for $E>10 \mathrm{MeV}$ as a function of the flare location (left) and the CME angular width (right). The data points with a width of $360^{\circ}$ correspond to halo CMEs. The correlation coefficient $\mathrm{r}$ and its uncertainty is also shown.

Table 1. The correlation coefficients between the logarithm of the proton peak flux (for $E>10 \mathrm{MeV}$ and $E>60 \mathrm{MeV}$ ) and the following characteristics of the associated solar eruptive events: logarithm of flare intensity $\log \left(I_{\mathrm{f}}\right)$, flare longitude $L_{\mathrm{f}}, \mathrm{CME}$ speed $v_{\mathrm{CME}}$ and $\mathrm{CME}$ angular width $w_{\mathrm{CME}}$. The correlations with flare $(\mathrm{CME})$ parameters are based on 88 (78) events.

\begin{tabular}{ccccc}
\hline & $\log \left(I_{\mathrm{f}}\right)$ & $L_{\mathrm{f}}$ & $v_{\mathrm{CME}}$ & $w_{\mathrm{CME}}$ \\
\hline$E>10 \mathrm{MeV}$ & $0.55 \pm 0.07$ & $0.12 \pm 0.11$ & $0.56 \pm 0.08$ & $0.16 \pm 0.12$ \\
$E>60 \mathrm{MeV}$ & $0.63 \pm 0.06$ & $0.19 \pm 0.11$ & $0.40 \pm 0.09$ & $0.13 \pm 0.12$ \\
\hline
\end{tabular}

between the peak flux in the lowest energy channel $(5.00-7.23 \mathrm{MeV})$ and the CME speed. The negligible effect on the correlations is not surprising as only half of the events in the SSE list contain an identified ESP, and often the secondary flux enhancement due to the shock passage does not exceed the initial peak flux. This is in particular the case for higher energy channels.

As Figures 15 17 and Table 1 indicate, the strongest correlations exist between the proton peak flux and flare intensity and CME speed. To provide a clearer quantification of the dependency of the peak flux on the solar parameters which could be used within a forecasting framework, we adopted a binning of the data points of Figures 15 and 16. Also for the analysis of the peak fluxes, the binning has been chosen such that they contain sufficient events and also taking into account the shape of the distributions. The logarithm of the flare intensity was divided in four equidistant bins between -5.0 and $-3.3 \log (\mathrm{W}$ $\mathrm{m}^{-2}$ ), while all events with larger values were collected in one bin, resulting in the following binning: $[-5.0,-4.6],[-4.6,-4.2],[-4.2,-3.8],[-3.8,-3.3],[>-3.3]$ in units of $\log \left(\mathrm{W} \mathrm{m}^{-2}\right)$. In terms of flare magnitude, this corresponds to the bins [M1.0,M2.5], [M2.5,M6.3], [M6.3,X1.6], [X1.6,X5.0] and [ $\geq$ X5.0]. The CME speed was binned into three equidistant bins between 1000 and $2200 \mathrm{~km} \mathrm{~s}^{-1}$, and the events associated to slower as well as faster CMEs were grouped in one bin each, giving the following bins: [400, 1000], [1000, 1400], [1400, 1800], [1800, 2200], 


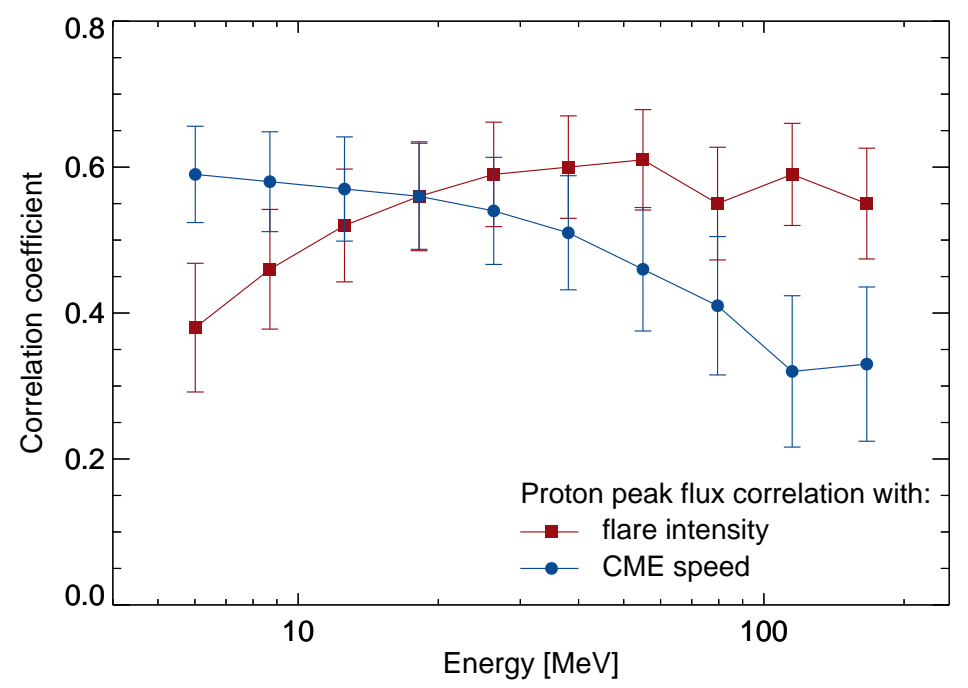

Figure 18. The correlation coefficients between the proton peak flux and the flare intensity (red squares) and CME speed (blue circles) as a function of the proton energy.

Table 2. The correlation coefficients between the logarithm of the proton peak flux as measured in each SEPEM energy channel and the logarithm of flare intensity $\log \left(I_{\mathrm{f}}\right)$ and CME speed $v_{\mathrm{CME}}$, excluding and including the ESP contribution in the flux profile.

\begin{tabular}{ccccc}
\hline \multirow{2}{*}{$\begin{array}{c}\text { SEPEM proton } \\
\text { energy channel }\end{array}$} & \multicolumn{2}{c}{ ESP excluded } & \multicolumn{2}{c}{ ESP included } \\
& $\log \left(I_{\mathrm{f}}\right)$ & $v_{\mathrm{CME}}$ & $\log \left(I_{\mathrm{f}}\right)$ & $v_{\mathrm{CME}}$ \\
\hline $5.00-7.23 \mathrm{MeV}$ & $0.38 \pm 0.09$ & $0.59 \pm 0.06$ & $0.41 \pm 0.09$ & $0.52 \pm 0.07$ \\
$7.23-10.46 \mathrm{MeV}$ & $0.46 \pm 0.08$ & $0.58 \pm 0.07$ & $0.46 \pm 0.08$ & $0.56 \pm 0.07$ \\
$10.46-15.12 \mathrm{MeV}$ & $0.52 \pm 0.08$ & $0.57 \pm 0.07$ & $0.50 \pm 0.08$ & $0.57 \pm 0.07$ \\
$15.12-21.87 \mathrm{MeV}$ & $0.56 \pm 0.07$ & $0.56 \pm 0.07$ & $0.54 \pm 0.08$ & $0.56 \pm 0.07$ \\
$21.87-31.62 \mathrm{MeV}$ & $0.59 \pm 0.07$ & $0.54 \pm 0.07$ & $0.57 \pm 0.07$ & $0.55 \pm 0.07$ \\
$31.62-45.73 \mathrm{MeV}$ & $0.60 \pm 0.07$ & $0.51 \pm 0.08$ & $0.59 \pm 0.07$ & $0.52 \pm 0.07$ \\
$45.73-66.13 \mathrm{MeV}$ & $0.61 \pm 0.07$ & $0.46 \pm 0.09$ & $0.60 \pm 0.07$ & $0.46 \pm 0.08$ \\
$66.13-95.64 \mathrm{MeV}$ & $0.55 \pm 0.08$ & $0.41 \pm 0.09$ & $0.55 \pm 0.08$ & $0.41 \pm 0.09$ \\
$95.64-138.3 \mathrm{MeV}$ & $0.59 \pm 0.07$ & $0.32 \pm 0.10$ & $0.60 \pm 0.07$ & $0.33 \pm 0.10$ \\
$138.3-200.0 \mathrm{MeV}$ & $0.55 \pm 0.08$ & $0.33 \pm 0.11$ & $0.53 \pm 0.07$ & $0.32 \pm 0.10$ \\
\hline
\end{tabular}

[>2200] in units of $\mathrm{km} \mathrm{s}^{-1}$. The lower limit in the first bin is only due to the absence of SEP events associated to CMEs slower than $400 \mathrm{~km} \mathrm{~s}^{-1}$. The mean of the logarithm of the peak fluxes is then calculated from the data points that fall within each bin. As an estimate of the spread of the events within a bin, the root mean square (RMS) of the values in each bin is calculated. The median, $25 \%$ and $75 \%$ quartiles, and extreme values are also determined. Figures 19 and 20. show the binned data for the integrated flux at $E>10 \mathrm{MeV}$ and $E>60 \mathrm{MeV}$ versus flare intensity and CME speed respectively. As was already evident from the scatter plots, there is a clear dependence of the peak fluxes on both flare 

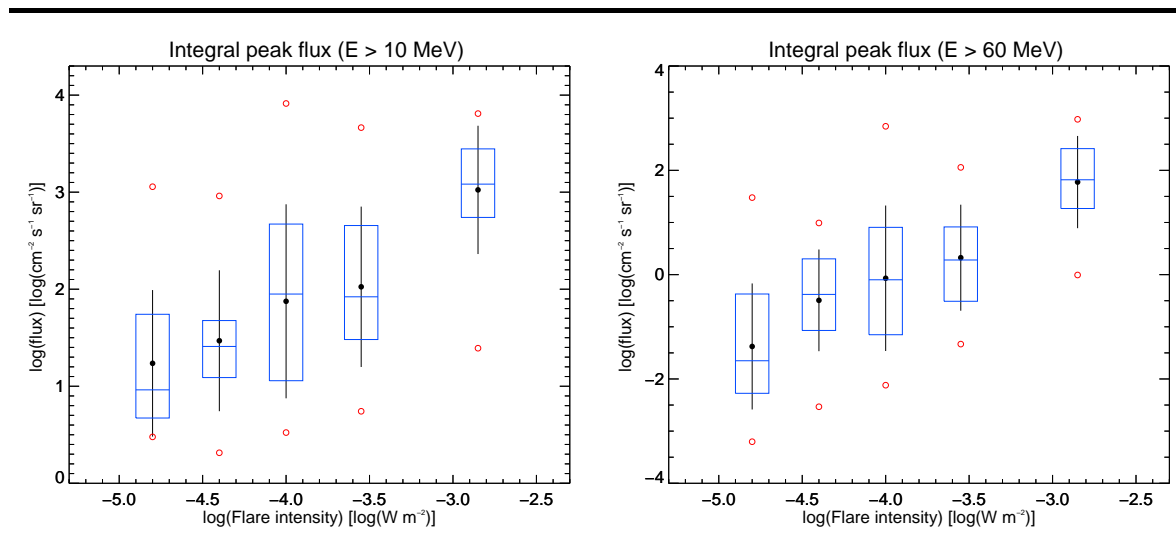

Figure 19. Binned plots of the logarithm of the proton peak flux as a function of logarithm of flare intensity for $E>10 \mathrm{MeV}$ (left) and $E>60 \mathrm{MeV}$ (right). Black dots show the mean in that bin, while the error bars represent the RMS. The horizontal blue line shows the median, and the box shows the start of the second quartile and end of the third quartile, so that $50 \%$ of the events lie in the box. Red open circles show the outermost data points (outliers) in each bin.
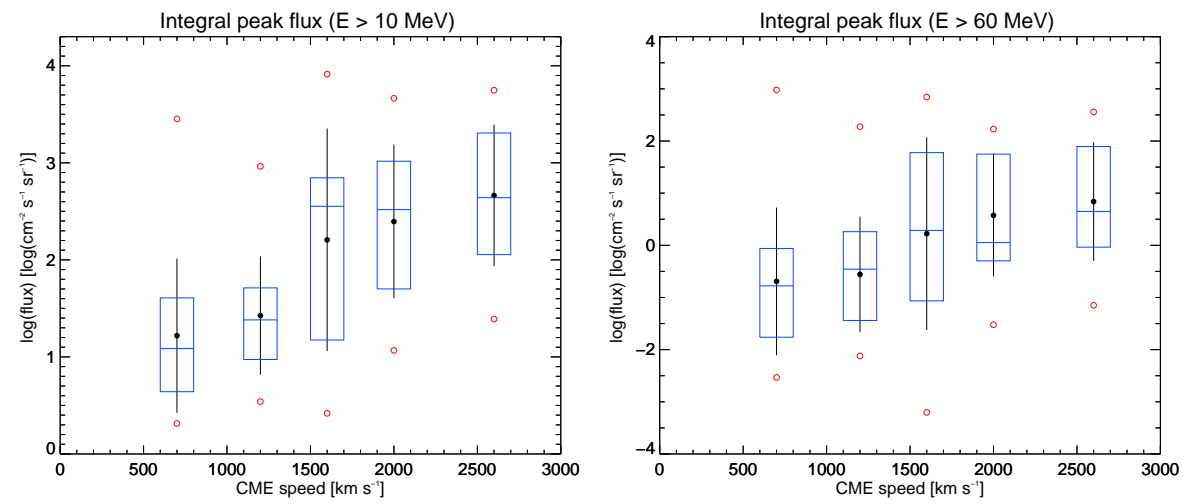

Figure 20. Binned plots of the logarithm of the proton peak flux as a function of the CME speed for $E>10 \mathrm{MeV}$ (left) and $E>60 \mathrm{MeV}$ (right). The convention for the plot is the same as in Figure 19

intensity and CME speed. Tables 12 and 13 in the Appendix give values of the mean and RMS in each bin as a function of flare intensity and CME speed respectively, for the peak fluxes in the two energy ranges considered.

\subsubsection{Peak Flux as a Function of Multiple Flare and CME Parameters}

We study the dependency of the proton peak fluxes on several combinations of flare and CME parameters by dividing one of the parameters under study into bins and determining in each bin the correlation between the integral proton peak flux (for $E>10 \mathrm{MeV}$ and $E>60 \mathrm{MeV}$ ) and the other solar parameters. Subsequently, the second solar parameter is also binned and the mean, RMS, quartiles and extremes in each bin are determined. Only a limited amount of bins is used due to the low statistics. The M-class flares were binned in two bins 
Table 3. Correlation coefficients of the peak flux of protons with $E>10 \mathrm{MeV}$ (top) and $E>60 \mathrm{MeV}$ (bottom) with the solar parameters given in the first column in different bins of flare magnitude $I_{\mathrm{f}}$, flare longitude location $L_{\mathrm{f}}$ and CME speed $v_{\mathrm{CME}}$. The number of events used to derive each correlation coefficient is given in brackets.

\begin{tabular}{|c|c|c|c|}
\hline \multicolumn{4}{|c|}{ proton peak flux $E>10 \mathrm{MeV}$} \\
\hline flare longitude & $\begin{aligned} \mathrm{M} 1.0 & \leq I_{\mathrm{f}}<\mathrm{M} 3.2 \\
0.38 & \pm 0.21(20)\end{aligned}$ & $\begin{array}{c}\mathrm{M} 3.2 \leq I_{\mathrm{f}}<\mathrm{X} 1.0 \\
0.30 \pm 0.14(30)\end{array}$ & $\begin{array}{c}I_{\mathrm{f}} \geq \mathrm{X} 1.0 \\
0.01 \pm 0.15(38)\end{array}$ \\
\hline CME speed & $0.59 \pm 0.22(15)$ & $0.40 \pm 0.12(28)$ & $0.61 \pm 0.10(35)$ \\
\hline CME width & $0.16 \pm 0.20(15)$ & $0.17 \pm 0.20(28)$ & $-0.02 \pm 0.20(35)$ \\
\hline $\begin{array}{l}\text { flare intensity } \\
\text { CME speed }\end{array}$ & $\begin{array}{c}-90^{\circ} \leq L_{\mathrm{f}}<-30^{\circ} \\
0.72 \pm 0.29(7) \\
0.27\end{array}$ & $\begin{array}{c}-30^{\circ} \leq L_{\mathrm{f}}<30^{\circ} \\
0.62 \pm 0.12(38) \\
0.72 \pm 0.07(35)\end{array}$ & $\begin{array}{l}30^{\circ} \leq L_{\mathrm{f}} \leq 90^{\circ} \\
0.52 \pm 0.10(43) \\
0.45 \pm 0.12(39)\end{array}$ \\
\hline CME width & $\begin{array}{c}v_{\mathrm{CME}}<1500 \mathrm{~km} \mathrm{~s}^{-1} \\
-0.03 \pm 0.15(42)\end{array}$ & $\begin{array}{c}v_{\mathrm{CME}} \geq 1500 \mathrm{~km} \mathrm{~s}^{-1} \\
0.28 \pm 0.15(36)\end{array}$ & \\
\hline \multicolumn{4}{|c|}{ proton peak flux $E>60 \mathrm{MeV}$} \\
\hline $\begin{array}{l}\text { flare longitude } \\
\text { CME speed } \\
\text { CME width }\end{array}$ & $\begin{array}{c}\mathrm{M} 1.0 \leq I_{\mathrm{f}}<\mathrm{M} 3.2 \\
0.40 \pm 0.25(20) \\
0.25 \pm 0.36(15) \\
0.06 \pm 0.22(15)\end{array}$ & $\begin{array}{c}\mathrm{M} 3.2 \leq I_{\mathrm{f}}<\mathrm{X} 1.0 \\
0.44 \pm 0.13(30) \\
0.27 \pm 0.16(28) \\
0.05 \pm 0.21(28)\end{array}$ & $\begin{array}{c}I_{\mathrm{f}} \geq \mathrm{X} 1.0 \\
0.12 \pm 0.14(23) \\
0.38 \pm 0.17(38) \\
0.01 \pm 0.20(38)\end{array}$ \\
\hline $\begin{array}{l}\text { flare intensity } \\
\text { CME speed }\end{array}$ & $\begin{array}{c}-90^{\circ} \leq L_{\mathrm{f}}<-30^{\circ} \\
0.90 \pm 0.11^{\dagger}(7) \\
0.54^{\ddagger}(4)\end{array}$ & $\begin{array}{c}-30^{\circ} \leq L_{\mathrm{f}}<30^{\circ} \\
0.73 \pm 0.09(38) \\
0.63 \pm 0.09\end{array}$ & $\begin{array}{l}30^{\circ} \leq L_{\mathrm{f}} \leq 90^{\circ} \\
0.60 \pm 0.09(43) \\
0.25 \pm 0.14(39)\end{array}$ \\
\hline CME width & $\begin{array}{c}v_{\mathrm{CME}}<1500 \mathrm{~km} \mathrm{~s}^{-1} \\
-0.02 \pm 0.14(42)\end{array}$ & $\begin{array}{c}v_{\mathrm{CME}} \geq 1500 \mathrm{~km} \mathrm{~s}^{-1} \\
0.22 \pm 0.19(36)\end{array}$ & \\
\hline
\end{tabular}

equidistant in the logarithm of the flare intensity, while one bin was used for $\mathrm{X}$-class flares, resulting in the following flare magnitude bins: $\mathrm{M} 1 \leq I_{\mathrm{f}}<\mathrm{M} 3.2$, $\mathrm{M} 3.2 \leq I_{\mathrm{f}}<\mathrm{X} 1$ and $I_{\mathrm{f}} \geq \mathrm{X} 1$. For the flare longitude three equidistant bins were chosen: $-90^{\circ} \leq L_{\mathrm{f}}<-30^{\circ}$ (east), $-30^{\circ} \leq L_{\mathrm{f}}<30^{\circ}$ (center) and $30^{\circ} \leq$ $L_{\mathrm{f}}<90^{\circ}$ (west). The CME speed is binned in two equal sized bins for slower CMEs and one bin for fast CMEs: $400 \leq v_{\mathrm{CME}}<1200,1200 \leq v_{\mathrm{CME}}<2000$ and $v_{\mathrm{CME}} \geq 2000$ in units of $\mathrm{km} \mathrm{s}^{-1}$, while the width is divided into the bins $0^{\circ} \leq w_{\mathrm{CME}}<100^{\circ}, 100^{\circ} \leq w_{\mathrm{CME}}<360^{\circ}$ and $w_{\mathrm{CME}}=360^{\circ}$. The latter bin corresponds to halo CMEs. All derived correlation coefficients for the different combinations of parameters are presented in Table 3 and further described below.

The correlation coefficient between the peak flux with $E>10 \mathrm{MeV}$ and flare longitude shows a weak correlation for the two M-class flare bins $(0.38 \pm 0.21$ and $0.30 \pm 0.14)$, while the correlation is non-existent for X-class flares $(0.01 \pm 0.15)$. This difference is quite similar for the integral peak flux above $60 \mathrm{MeV}$ : a weak to moderate correlation with longitude $(0.40 \pm 0.25$ and $0.44 \pm 0.13)$ for Mclass flares, and consistent with zero for the X-class flares $(0.12 \pm 0.14)$. The mean peak fluxes in each of the bins for the combination of flare magnitude and 

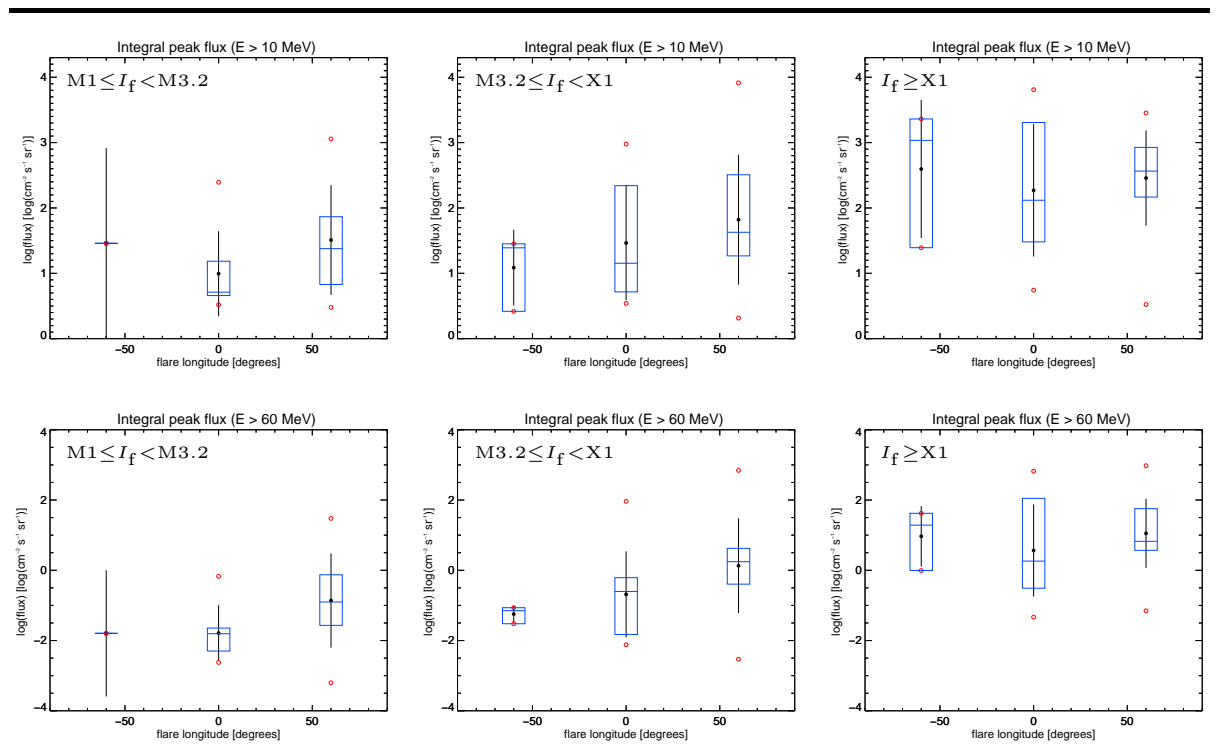

Figure 21. Binned plots of the logarithm of the peak flux for $E>10 \mathrm{MeV}$ (top) and $E>60 \mathrm{MeV}$ protons (bottom) as a function of the longitude of the flare location for three different flare magnitude bins: $\mathrm{M} 1 \leq I_{\mathrm{f}}<\mathrm{M} 3.2$ (left), M3.2 $\leq I_{\mathrm{f}}<\mathrm{X} 1$ (middle) and $I_{\mathrm{f}} \geq \mathrm{X} 1$ (right). The convention the plot is the same as in Figure 19

longitude are shown in Figure 21 and listed in Table 14 in the Appendix. The correlations between the peak flux and flare intensity for the three longitude bins are also listed in Table 3 and show no significant dependency on longitude for $E>10 \mathrm{MeV}$ moving from the east towards the west side of the Sun $(0.72 \pm 0.29$, $0.62 \pm 0.12$ and $0.52 \pm 0.10)$, but a significant decrease for $E>60 \mathrm{MeV}(0.90 \pm 0.11$, $0.73 \pm 0.09$ and $0.60 \pm 0.09)$. It should be noted that the easternmost bin merely contains seven events.

The correlations have also been derived for the three flare magnitude and longitude bins between the proton peak fluxes and the CME speed. The correlation with the CME speed for $E>10 \mathrm{MeV}$ increases from $0.40 \pm 0.12$ for the stronger M-class flares to $0.61 \pm 0.10$ for X-class flares. The behavior towards smaller flare intensities is unclear due to the relatively large uncertainty $(0.59 \pm 0.22)$. The difference is not significant for the $E>60 \mathrm{MeV}$ peak fluxes: changing from $0.25 \pm 0.36$ and $0.27 \pm 0.16$ for the two M-class flare bins to $0.38 \pm 0.17$ for the $\mathrm{X}$-class flares. The mean peak fluxes in each of the bins for the combination of flare peak intensity and CME speed are shown in Figure 22 and listed in Table15 in the Appendix. Note that there are no SEP events present in the data sample associated with a flare of magnitude weaker than M3.2 and a CME with a speed faster than $2000 \mathrm{~km} \mathrm{~s}^{-1}$. Table 3 shows that for both energy channels, the correlation with the CME speed is the largest for the central location $(0.72 \pm 0.07$ and $0.63 \pm 0.09)$ and is significantly smaller for longitudes west of $30^{\circ}(0.45 \pm 0.12$ and $0.25 \pm 0.14)$. The easternmost bin contains only four events.

Finally, the dependence of the peak fluxes on the CME width has been determined in the three flare magnitude and two CME speed bins. The correlations between the peak flux for both energy ranges and the CME width remains within 

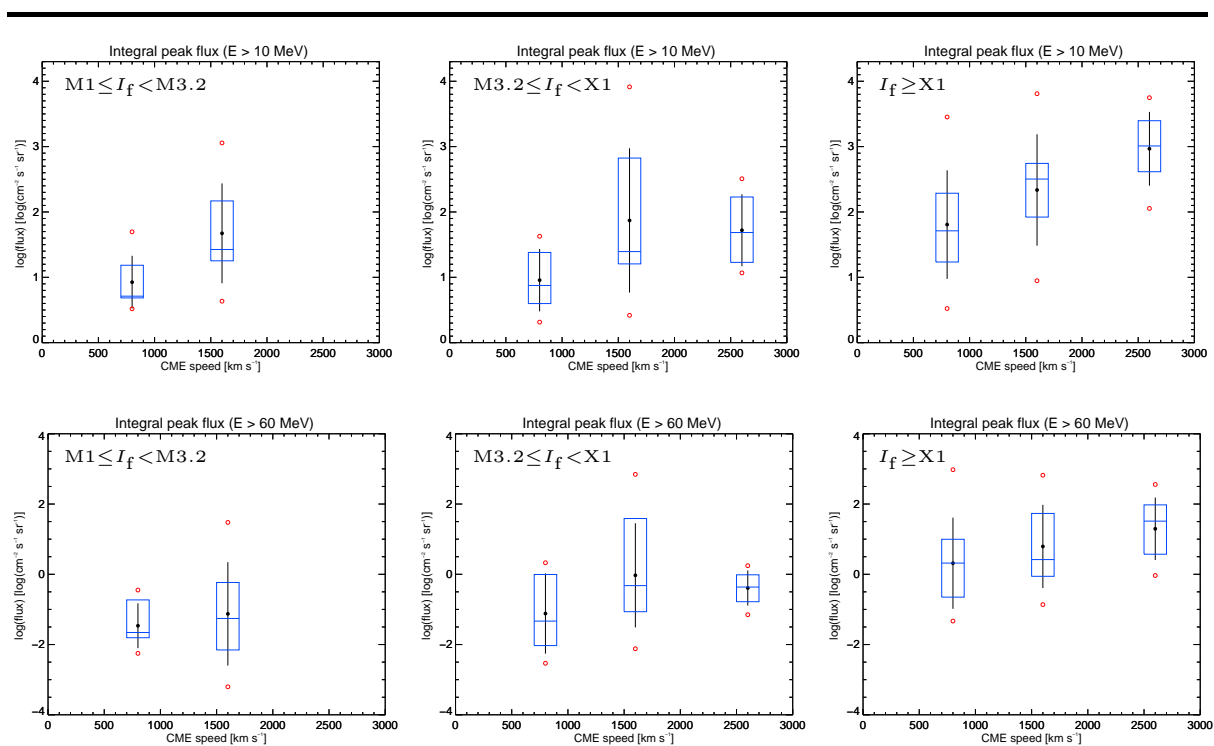

Figure 22. Binned plots of the logarithm of the peak flux for $E>10 \mathrm{MeV}$ (top) and $E>60 \mathrm{MeV}$ protons (bottom) as a function of the CME speed for three different flare magnitude bins: M1 $\leq I_{\mathrm{f}}<\mathrm{M} 3.2$ (left), M3.2 $\leq I_{\mathrm{f}}<\mathrm{X} 1$ (middle) and $I_{\mathrm{f}} \geq \mathrm{X} 1$ (right) The convention for the plot is the same as in Figure 19

the error close to zero regardless of the flare magnitude bin. Figure 23 shows the mean peak flux in each bin when flare intensity and CME width are combined and these values are listed in Table 16 in the Appendix. The correlation with the CME width has been derived in two bins of CME speed, namely smaller or greater than $1500 \mathrm{~km} \mathrm{~s}^{-1}$, and are given in Table 3 For both energy ranges, there exists no correlation for slow CMEs $(-0.03 \pm 0.15$ for $E>10 \mathrm{MeV}$ and $-0.02 \pm 0.14$ for $E>60 \mathrm{MeV}$ ), while a very weak correlation exists for fast CMEs $(0.28 \pm 0.15$ for $E>10 \mathrm{MeV}$ and $0.22 \pm 0.19$ for $E>60 \mathrm{MeV})$. The mean peak fluxes in each of the bins for the combination of the CME speed and width are shown in Figure 24 and listed in Table 17 in the Appendix.

\section{Discussion and Conclusions}

We have reported on the results of a statistical analysis of the dependence of SEP occurrence probability and proton peak fluxes at $1 \mathrm{AU}$ on both flare (intensity and longitude) and CME (speed and angular width) characteristics. The work presented here goes beyond the usual study of the relationship between the SEP event and the individual solar parameters by investigating the effect of combining these parameters. Furthermore, the energy dependence of the correlation between the proton peak flux and the solar parameters was derived and the influence of the ESPs on these correlations was explored.

The results presented here show that the SEP occurrence probabilities generally increase significantly for events associated with large X-class flares, western solar longitudes, CMEs with high velocities and halo CMEs. It is found that 

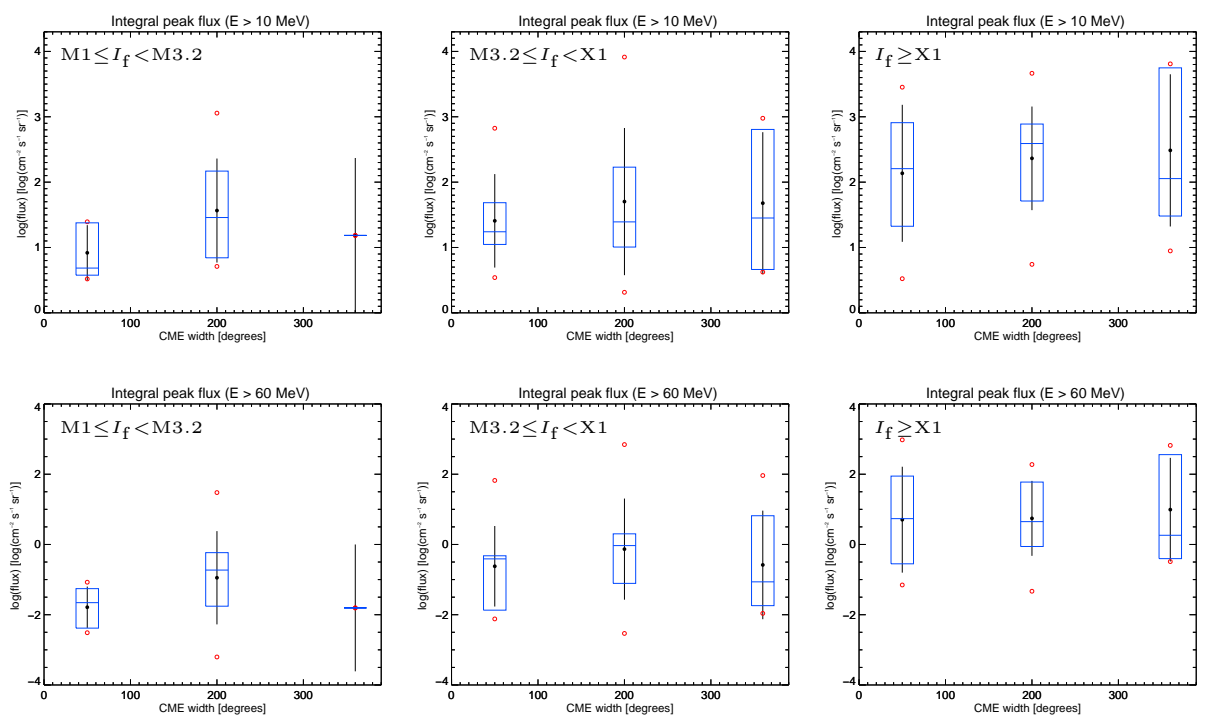

Figure 23. Binned plots of the logarithm of the peak flux for $E>10 \mathrm{MeV}$ (top) and $E>60 \mathrm{MeV}$ protons (bottom) as a function of the CME width for three different flare magnitude bins: $M 1 \leq I_{\mathrm{f}}<M 3.2$ (left), $M 3.2 \leq I_{\mathrm{f}}<X 1$ (middle) and $I_{\mathrm{f}} \geq X 1$ (right). The convention for the plot is the same as in Figure 19
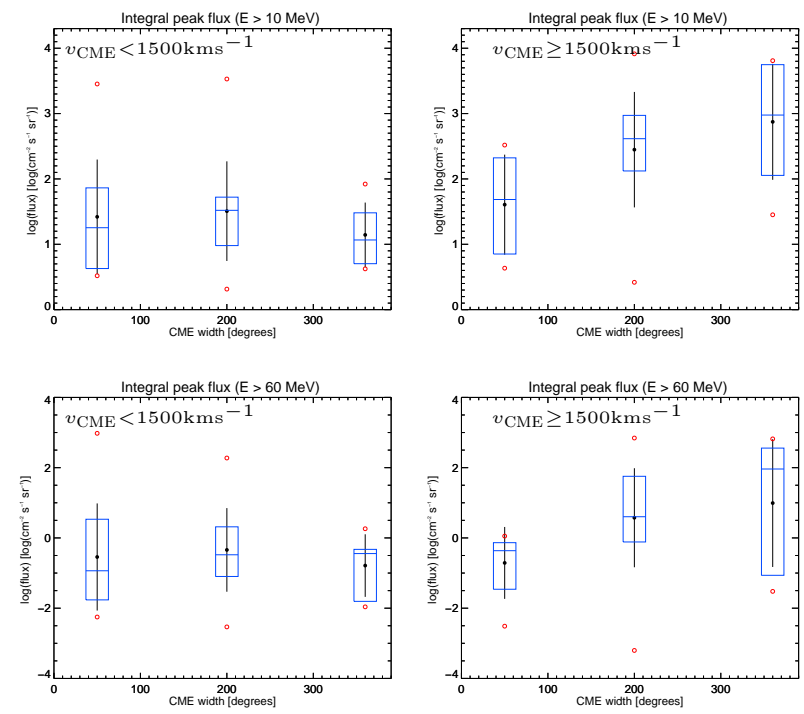

Figure 24. Binned plots of the logarithm of the peak flux for $E>10 \mathrm{MeV}$ (top) and $E>60 \mathrm{MeV}$ protons (bottom) as a function of the CME width for two different CME speed bins: $v_{\mathrm{CME}}<1500 \mathrm{~km} \mathrm{~s}^{-1}$ (left), and $v_{\mathrm{CME}} \geq 1500 \mathrm{~km} \mathrm{~s}^{-1}$ (right). The convention for the plot is the same as in Figure 19 
the proton peak fluxes increase with flare intensity and CME speed as well, but exhibit little correlation with the flare longitude and CME width. This is in general agreement with previous works which will be discussed in detail below. The observed qualitative behavior of the SEP occurrence probability is similar irrespective of the data set used for the derivation of these probabilities. The results obtained with the CRR2010 and SSE lists show some small differences in the actual probability values; this is a consequence of the different energy ranges used, and of the presence of electron dominated SEP events in the CRR2010 list that are excluded from the SSE list. This demonstrates that the selection criteria for SEP events have an impact on the derived probability values and that it is important that these criteria are documented in detail.

We have determined the correlation coefficients between the proton peak flux and the solar parameters for each individual SEPEM energy channel (Figure 18). The correlation with flare intensity increases with energy, while the correlation with CME speed shows the opposite behavior. Furthermore, the correlation with the CME speed is stronger than the correlation with the flare intensity below $10 \mathrm{MeV}$ and is weaker above $50 \mathrm{MeV}$. While the correlation coefficients derived here do not single out a specific acceleration mechanism, they do give an idea of the origin of the energetic particles. The two correlations coefficients are of similar size in the intermediate energy range which indicates a mixed-SEP origin where both flare and CME shock driven acceleration contribute as proposed by Kallenrode (2003) and supported by the recent work of Trottet et al. (2014). Under this assumption, our results suggest that acceleration near the flare site, or at least the conditions that lead to stronger flares, are more important for the production of protons at higher energies than CME related acceleration, while the situation seems opposite for less energetic protons. This conclusion supports earlier findings based on data (see references in Trottet et al. (2014)). Additionally, a recent model was developed allowing for the simultaneous existence of acceleration in the corona as the main source for protons $>50 \mathrm{MeV}$ together with CME-driven shock acceleration dominating at lower proton energies (Kocharov et al., 2014). While the energy dependence deserves a more detailed study including more data, this behavior is an important observation that needs to be taken into account when comparing studies based on different proton energy ranges. It also provides valuable information for the development of space weather forecasts in regard to the effects resulting from low and high energy protons.

We observe a strong rise in both the probability of SEP occurrence (Figure 4 and Table 5 in the Appendix) and mean proton peak fluxes (Figure 19 and Table 12 in the Appendix) with increasing flare intensities. These results are quantitatively and qualitatively similar to those of Belov et al. (2005) (their Figures 10 and 12). The results from Park et al. (2010) show the same behavior, but the actual probabilities differ significantly (their Table 1). Their derived correlation coefficient between the peak flux for $E>10 \mathrm{MeV}$ and the flare intensity is 0.43 , while we obtained $0.55 \pm 0.07$. We believe this difference is due to a difference in the criteria for SEP event selection and for solar event association. While their flare sample is almost three times larger than considered here due to the larger period covered (1976 to 2006), the number of SEP events are very 
similar to ours (166 compared to 160 in the CRR2010 and 90 in the SSE list). The correlation coefficient presented here is in agreement with the value derived by Cane, Richardson, and von Rosenvinge (2010). Our result is much larger than the value determined by Hwang et al. (2010) (0.37). They only analyzed SEP events that have both associated CMEs and flares, while we do not require the presence of a CME to derive this correlation. Furthermore, their sample includes flares originating beyond the limb which reduces the correlation with the proton peak flux as the observed flare intensity is underestimated. Cliver et al. (2012) derived a value of 0.52 for events originating from longitudes between west $20^{\circ}$ to $85^{\circ}$ and our result is in very good agreement when limited to the same region $(0.52 \pm 0.10)$. Our value is also in very good agreement with the correlation coefficient of $0.59 \pm 0.07$ obtained by Miteva et al. (2013). Using proton peak fluxes around $25 \mathrm{MeV}$ from events originating on the western front-side of the Sun with respect to the observing instrument, Richardson et al. (2014) found a value (0.71) which is larger than ours. This small difference could be the consequence of the time period they analyzed (2009 to 2012) and of including C and B flares.

Our analysis shows that the probability for SEP occurrence grows significantly when the flare occurs more towards the western side of the Sun (Figure 5 and Table 6 in the Appendix), while the proton peak fluxes show almost no correlation with longitude (Figure 17 and Table 11). Our results are in agreement with Belov et al. (2005) which demonstrated that the SEP occurrence probability is remarkably high for flares westward of $70^{\circ}$. Our derived probabilities as a function of longitude are comparable with the longitudinal distribution of SEPassociated flare fractions (Figure 8 of their paper) in the M8 to X3 flare strength range. In an earlier analysis by the same authors (Kurt et al., 2004), an order of magnitude difference was observed in the mean peak flux of protons with $E>10 \mathrm{MeV}$ between events originating from longitudes smaller and larger than $-30^{\circ}$ (their Figure 6). Comparing with Figure 17, our results do not confirm this difference due to the low statistics of events with longitude $<-30^{\circ}$. Considering events associated with solar flares located at longitudes $>-30^{\circ}$, they observe an average peak flux of around $1000 \mathrm{~cm}^{-2} \mathrm{~s}^{-1} \mathrm{sr}^{-1}$, while most of our events are below this value. Our observation on the dependence of the probability on flare longitude shows a good qualitative agreement with the result from Park et al. (2010) (their Figure 1), but also in this case their actual values differ significantly from ours. Several studies have investigated the dependence of probability of SEP occurrence on longitude by using the source location of the associated CME (Gopalswamy et al., 2008; Park, Moon, and Gopalswamy, 2012) and also concluded that a larger fraction of events originating at western longitudes result in SEP events.

We determined that the CME speed has a strong influence on the probability, regardless of flare intensity (Figures 9 and 12, and Tables 8 and 11 in the Appendix), and on the mean peak fluxes (Figure 20 and Table 13 in the Appendix). Although different selection criteria were used, the large increase of the probability with the CME speed was also observed by Gopalswamy et al. (2008) and Park, Moon, and Gopalswamy (2012). The latter authors also derived a correlation coefficient between the CME speed and the proton peak flux of 0.57. 
Our value $(0.56 \pm 0.08$ for $E>10 \mathrm{MeV})$ agrees well with this result, as well as with those from Cane, Richardson, and von Rosenvinge (2010) (about 0.6) and Miteva et al. (2013) $(0.63 \pm 0.05$, although based on the logarithm of the CME speed). As was the case for the correlation with flare intensity, Hwang et al. (2010) measured a much smaller value (0.31) and Richardson et al. (2014) a significantly larger value $(0.66-0.71)$ compared to ours.

From Figure 12 (and Table 11 in the Appendix) it is clear that the SEP occurrence probability is significantly higher for halo CMEs compared to non-halo CMEs, in qualitative agreement with the conclusions from Gopalswamy et al. (2008) and Park, Moon, and Gopalswamy (2012). We observed no dependence of the proton peak fluxes on the CME width (Figure 17 and Table 1) and derived a correlation coefficient of $0.16 \pm 0.12$ (for $E>10 \mathrm{MeV}$ ). This is slightly smaller than the value observed by Miteva et al. (2013) (0.29 \pm 0.12$)$. Cane, Richardson, and von Rosenvinge (2010) showed the relation between the proton peak flux and the CME width (their Figure 10) without quantifying this correlation, and no strong dependence was observed which our results also indicate. Park et al. (2010) looked at the correlation for partial and full halo CMEs separately, finding no evidence for a correlation between the proton peak flux and the CME width, a conclusion also supported by our results.

Several studies have already investigated the dependence of SEP probabilities and peak fluxes on multiple solar parameters, but were limited to a few combinations of two parameters or derived the dependence on one variable in the specific range of another parameter. In this work, we derived the SEP occurrence probability taking fully into account both flare and CME characteristics. Figures 8,11 (and their respective Tables 7,10 in the Appendix) clearly indicate a differentiation of SEP occurrence probability when all flare (location and intensity) and CME (velocity and width) characteristics are taken into account with respect to probabilities derived from a single parameter or combinations of specific source characteristics. Differences in results from the single parameter and multi-dimensional analysis are expected, as the SEP occurrence probabilities rely on the characteristics of events at the source of the SEPs (flare and CMEs). This conclusion also holds for the dependency of the proton peak fluxes on the solar parameters as can be seen from Figures 21]24 (and Tables 14] 17 in the Appendix).

The correlation coefficients between the proton peak flux and flare longitude in different flare magnitude ranges for $E>10 \mathrm{MeV}$ are weak for the two M flare bins $(0.29 \pm 0.25$ and $0.30 \pm 0.14)$ but remain consistent with zero for the $\mathrm{X}$ flares $(0.01 \pm 0.15)$. The difference is even larger for the $E>60 \mathrm{MeV}$ range, where the correlations are moderate for $\mathrm{M}$ flares $(0.52 \pm 0.19$ and $0.44 \pm 0.13)$ but again non-existent for $\mathrm{X}$ flares $(0.12 \pm 0.14)$. The correlation with the CME speed also shows a dependency on flare magnitude: it increases from $0.40 \pm 0.12$ for M flares $\geq$ M3.2 to $0.61 \pm 0.10$ for X flares for $E>10 \mathrm{MeV}$. This is consistent with the value of 0.69 that Park, Moon, and Gopalswamy (2012) derived for X flares. The increase is not significant for $E>60 \mathrm{MeV}$ (from $0.27 \pm 0.16$ to $0.38 \pm 0.17$ ). The uncertainties on the correlation coefficients for the weaker $\mathrm{M}$ flares are too large to confirm this dependency towards smaller flares. Within the uncertainties, there is no indication of a dependency on the flare intensity of the correlations 
with the CME width. Park et al. (2010) claimed to observe a decrease of the correlation with the flare intensity going from eastern to western longitudes $(0.65,0.42,0.38)$, while Miteva et al. (2013) observed no significant change when restricting events to western longitudes (their Table 7). Our results do not show any significant dependency of the correlations on longitude for $E>10 \mathrm{MeV}$ $(0.72 \pm 0.29,0.62 \pm 0.12,0.52 \pm 0.10)$, while some decrease can be observed for the $E>60 \mathrm{MeV}$ energy range $(0.90 \pm 0.11,0.73 \pm 0.09,0.60 \pm 0.09)$. Park, Moon, and Gopalswamy (2012) observed a large difference between the correlations with the CME speed when the event originates from the central and western region (0.78 versus 0.47 ), while Miteva et al. (2013) did not observe a significant change when analyzing events from more western longitudes (their Table 7). Our derived values also show a significant decrease from $0.72 \pm 0.07(0.63 \pm 0.09)$ to $0.45 \pm 0.12(0.25 \pm 0.14)$ for $E>10 \mathrm{MeV}(E>60 \mathrm{MeV})$ going from central to western events.

For CMEs with a speed slower than $1500 \mathrm{~km} \mathrm{~s}^{-1}$, the correlation coefficients for both energy ranges remain consistent with zero, while the values increase for faster CMEs but are still weak.

When determining the proton peak flux during a SEP event, we have excluded any enhancements from the passage of an interplanetary shock wave through the observation point. For this purpose, lists of interplanetary shocks observed at the Wind and ACE spacecraft were used to identify ESP-like increases in the proton data. We have compared the derived correlation coefficients with the values obtained when the ESP contribution is not excluded, and found the differences to be negligible (see Table 21). This is not surprising, as only half of the events contain an ESP-like increase and the secondary enhancements often stay below the initial peak therefore not affecting the determination of the peak flux. Only the correlation between the peak flux in the lowest SEPEM energy channel (5.00$7.23 \mathrm{MeV}$ ) and the CME speed shows a small but not very significant decrease when the ESP contribution is included. It can be expected that the effect of the ESP on the correlation coefficients manifests itself mostly at the lower energies as the shock wave is more efficient at accelerating particles at lower energies.

In conclusion, our statistical analysis confirms results previously reported in the literature, and further expands our understanding of SEP occurrence probability and characteristics, since it involves a multi-parameter study based on both flare and CME characteristics. Furthermore, we observed an energy dependence of the correlation coefficient between the proton peak flux and both flare intensity and CME speed, and only observed a small but not very significant decrease in the lowest energy channel when excluding the ESP contribution to the flux. Derived results represent valuable input for SEP forecast tools and are incorporated within the Space Weather Alert System developed as part of the EU FP7 project COMESEP (Crosby et al. (2012), http://www.comesep.eu).

Acknowledgements This work has received funding from the European Union Seventh Framework Programme (FP7/2007-2013) under grant agreement n. 263252 [COMESEP]. We also acknowledge the ESA SEPEM reference proton dataset. 
Appendix

SOLA: sep_analysis.tex; 2 October 2018; 9:47; p. 28 
Table 4.: The SSE list and associated solar parameters as described in Section 2.2 containing the following information: the $\mathrm{SEP}$ onset date and time, the integral peak flux for $\mathrm{E}>10 \mathrm{MeV}$ and $\mathrm{E}>60 \mathrm{MeV}$, the flare magnitude and location, and the $\mathrm{CME}$ speed and width.

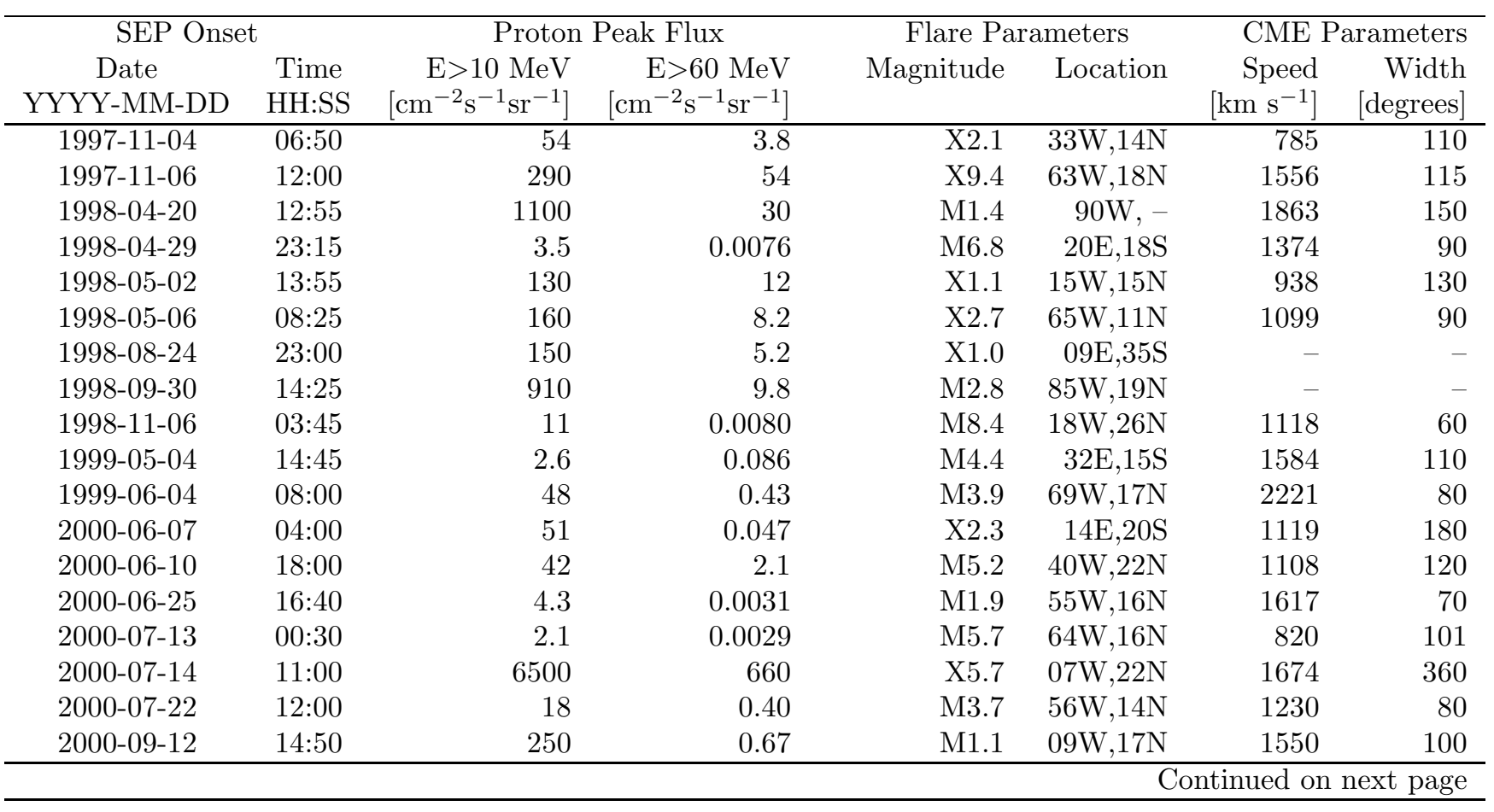


Table 4 - The SSE list and associated solar parameters (continued)

\begin{tabular}{|c|c|c|c|c|c|c|c|}
\hline \multicolumn{2}{|c|}{ SEP Onset } & \multicolumn{2}{|c|}{ Proton Peak Flux } & \multicolumn{2}{|c|}{ Flare Parameters } & \multicolumn{2}{|c|}{ CME Parameters } \\
\hline Date & Time & $\mathrm{E}>10 \mathrm{MeV}$ & $\mathrm{E}>60 \mathrm{MeV}$ & Magnitude & Location & Speed & Width \\
\hline YYYY-MM-DD & $\mathrm{HH}: \mathrm{SS}$ & {$\left[\mathrm{cm}^{-2} \mathrm{~s}^{-1} \mathrm{sr}^{-1}\right]$} & {$\left[\mathrm{cm}^{-2} \mathrm{~s}^{-1} \mathrm{sr}^{-1}\right]$} & & & {$\left[\mathrm{km} \mathrm{s}^{-1}\right]$} & [degrees] \\
\hline $2000-11-08$ & $23: 45$ & 8200 & 700 & M7.4 & $75 \mathrm{~W}, 10 \mathrm{~N}$ & 1738 & 120 \\
\hline $2000-11-24$ & 07:00 & 8.9 & 0.40 & $\mathrm{X} 2.0$ & $03 \mathrm{~W}, 22 \mathrm{~N}$ & 1289 & 360 \\
\hline 2000-11-24 & $16: 00$ & 84 & 1.8 & $\mathrm{X} 2.3$ & $07 \mathrm{~W}, 22 \mathrm{~N}$ & 1245 & 360 \\
\hline $2000-11-25$ & $18: 00$ & 25 & 0.071 & M8.2 & $50 \mathrm{E}, 07 \mathrm{~S}$ & 2519 & 120 \\
\hline 2001-01-22 & $01: 15$ & 3.0 & 0.033 & M1.1 & $32 \mathrm{~W}, 05 \mathrm{~N}$ & - & - \\
\hline 2001-01-28 & $19: 30$ & 50 & 0.36 & M1.5 & $59 \mathrm{~W}, 04 \mathrm{~N}$ & 916 & 120 \\
\hline 2001-03-29 & $13: 50$ & 30 & 0.33 & $\mathrm{X} 1.7$ & $12 \mathrm{~W}, 16 \mathrm{~N}$ & 942 & 360 \\
\hline 2001-04-02 & $11: 20$ & 3.3 & 0.070 & $\mathrm{X} 1.1$ & $62 \mathrm{~W}, 16 \mathrm{~N}$ & 992 & 50 \\
\hline 2001-04-02 & 23:00 & 770 & 18 & $\mathrm{X} 20$ & $78 \mathrm{~W}, 17 \mathrm{~N}$ & 2505 & 100 \\
\hline 2001-04-09 & $16: 00$ & 5.0 & 0.47 & M7.9 & $04 \mathrm{~W}, 21 \mathrm{~N}$ & 1192 & 360 \\
\hline 2001-04-10 & 07:00 & 110 & 0.92 & $\mathrm{X} 2.3$ & $09 \mathrm{~W}, 23 \mathrm{~N}$ & 2411 & 360 \\
\hline 2001-04-12 & $12: 00$ & 42 & 2.1 & $\mathrm{X} 2.0$ & $42 \mathrm{~W}, 20 \mathrm{~N}$ & 1184 & 120 \\
\hline 2001-04-14 & $18: 00$ & 1.6 & 0.0010 & M1.0 & $71 \mathrm{~W}, 17 \mathrm{~N}$ & 830 & 50 \\
\hline 2001-04-15 & $14: 00$ & 920 & 190 & $\mathrm{X} 14$ & $84 \mathrm{~W}, 20 \mathrm{~N}$ & 1199 & 110 \\
\hline 2001-04-27 & $04: 55$ & 4.2 & 0.011 & M7.8 & $31 \mathrm{~W}, 17 \mathrm{~N}$ & 1006 & 360 \\
\hline 2001-09-24 & $12: 00$ & 2000 & 78 & $\mathrm{X} 2.6$ & $23 \mathrm{E}, 16 \mathrm{~S}$ & 2402 & 120 \\
\hline 2001-10-09 & $14: 00$ & 5.1 & 0.019 & M1.4 & $10 \mathrm{E}, 25 \mathrm{~S}$ & 973 & 120 \\
\hline 2001-10-19 & $04: 55$ & 5.5 & 0.17 & $\mathrm{X} 1.6$ & $18 \mathrm{~W}, 16 \mathrm{~N}$ & 558 & 180 \\
\hline 2001-10-19 & $16: 00$ & 9.7 & 0.29 & X1.6 & $29 \mathrm{~W}, 15 \mathrm{~N}$ & 901 & 160 \\
\hline $2001-10-22$ & 04:00 & 0.14 & 0.00051 & M1.0 & $57 \mathrm{~W}, 17 \mathrm{~N}$ & 772 & 20 \\
\hline $2001-10-22$ & $15: 00$ & 23 & 0.80 & M6.7 & $18 \mathrm{E}, 21 \mathrm{~S}$ & 1336 & 140 \\
\hline 2001-11-04 & $16: 55$ & 950 & 92 & $\mathrm{X} 1.0$ & $18 \mathrm{~W}, 06 \mathrm{~N}$ & 1810 & 360 \\
\hline
\end{tabular}


Table 4 - The SSE list and associated solar parameters (continued)

\begin{tabular}{|c|c|c|c|c|c|c|c|}
\hline \multicolumn{2}{|c|}{ SEP Onset } & \multicolumn{2}{|c|}{ Proton Peak Flux } & \multicolumn{2}{|c|}{ Flare Parameters } & \multicolumn{2}{|c|}{ CME Parameters } \\
\hline $\begin{array}{c}\text { Date } \\
\text { YYYY-MM-DD }\end{array}$ & $\begin{array}{l}\text { Time } \\
\text { HH:SS }\end{array}$ & $\begin{array}{r}\mathrm{E}>10 \mathrm{MeV} \\
{\left[\mathrm{cm}^{-2} \mathrm{~s}^{-1} \mathrm{sr}^{-1}\right]}\end{array}$ & $\begin{array}{r}\mathrm{E}>60 \mathrm{MeV} \\
{\left[\mathrm{cm}^{-2} \mathrm{~s}^{-1} \mathrm{sr}^{-1}\right]}\end{array}$ & Magnitude & Location & $\begin{array}{r}\text { Speed } \\
{\left[\mathrm{km} \mathrm{s}^{-1}\right]}\end{array}$ & $\begin{array}{r}\text { Width } \\
\text { [degrees] }\end{array}$ \\
\hline 2001-11-17 & $19: 55$ & 29 & 0.016 & M2.8 & $42 \mathrm{E}, 13 \mathrm{~S}$ & 1379 & 160 \\
\hline 2001-11-22 & 21:00 & 19 & 2.0 & M3.8 & $67 \mathrm{~W}, 25 \mathrm{~N}$ & 1443 & 120 \\
\hline 2001-11-23 & 01:00 & 3400 & 39 & M9.9 & $34 \mathrm{~W}, 15 \mathrm{~N}$ & 1437 & 270 \\
\hline 2001-11-28 & $20: 00$ & 3.7 & 0.013 & M6.9 & $16 \mathrm{E}, 04 \mathrm{~S}$ & 500 & 90 \\
\hline $2001-12-26$ & $05: 55$ & 670 & 66 & M7.1 & $54 \mathrm{~W}, 08 \mathrm{~N}$ & 1446 & 90 \\
\hline 2002-03-16 & 08:05 & 15 & 0.016 & $\mathrm{M} 2.2$ & $03 \mathrm{~W}, 08 \mathrm{~N}$ & 957 & 360 \\
\hline 2002-03-22 & $12: 00$ & 14 & 0.00063 & M1.6 & $90 \mathrm{~W},-$ & 1750 & 130 \\
\hline 2002-04-17 & $11: 30$ & 23 & 0.084 & M2.6 & $34 \mathrm{~W}, 14 \mathrm{~N}$ & 1240 & 70 \\
\hline 2002-04-21 & 00:00 & 2300 & 60 & $\mathrm{X} 1.5$ & $84 \mathrm{~W}, 14 \mathrm{~N}$ & 2393 & 120 \\
\hline 2002-07-16 & $13: 20$ & 38 & 0.31 & $\mathrm{X} 3.0$ & $01 \mathrm{E}, 14 \mathrm{~S}$ & 1151 & 100 \\
\hline 2002-08-14 & 03:35 & 25 & 0.036 & M2.3 & $54 \mathrm{~W}, 09 \mathrm{~N}$ & 1309 & 60 \\
\hline 2002-08-16 & $22: 10$ & 5.4 & 0.016 & M5.2 & $20 \mathrm{E}, 14 \mathrm{~S}$ & 1585 & 160 \\
\hline $2002-08-18$ & 20:00 & 5.1 & 0.023 & M2.2 & $19 \mathrm{~W}, 12 \mathrm{~N}$ & 682 & 100 \\
\hline 2002-08-19 & $10: 00$ & 3.3 & 0.0056 & M2.0 & $25 \mathrm{~W}, 12 \mathrm{~N}$ & 549 & 80 \\
\hline 2002-08-22 & $03: 10$ & 33 & 2.1 & M5.4 & $62 \mathrm{~W}, 07 \mathrm{~N}$ & 998 & 80 \\
\hline 2002-08-24 & 00:00 & 340 & 34 & X3.1 & $81 \mathrm{~W}, 02 \mathrm{~N}$ & 1913 & 150 \\
\hline 2002-11-09 & $17: 10$ & 330 & 0.48 & M4.6 & $29 \mathrm{~W}, 12 \mathrm{~N}$ & 1838 & 90 \\
\hline 2003-05-28 & $07: 55$ & 12 & 0.14 & X3.6 & $20 \mathrm{~W}, 06 \mathrm{~N}$ & 1366 & 220 \\
\hline 2003-05-31 & 03:00 & 25 & 1.1 & M9.3 & $65 \mathrm{~W}, 07 \mathrm{~N}$ & 1835 & 150 \\
\hline 2003-06-18 & 20:00 & 28 & 0.030 & M6.8 & $55 \mathrm{E}, 07 \mathrm{~S}$ & 1813 & 360 \\
\hline 2003-10-23 & $00: 45$ & 4.6 & 0.0024 & M1.2 & $17 \mathrm{E}, 03 \mathrm{~S}$ & - & - \\
\hline 2003-10-26 & $18: 05$ & 510 & 3.6 & $\mathrm{X} 1.2$ & $38 \mathrm{~W}, 02 \mathrm{~N}$ & 1537 & 130 \\
\hline
\end{tabular}


Table 4 - The SSE list and associated solar parameters (continued)

\begin{tabular}{|c|c|c|c|c|c|c|c|}
\hline \multicolumn{2}{|c|}{ SEP Onset } & \multicolumn{2}{|c|}{ Proton Peak Flux } & \multicolumn{2}{|c|}{ Flare Parameters } & \multicolumn{2}{|c|}{ CME Parameters } \\
\hline $\begin{array}{c}\text { Date } \\
\text { YYYY-MM-DD }\end{array}$ & $\begin{array}{l}\text { Time } \\
\text { HH:SS }\end{array}$ & $\begin{array}{r}\mathrm{E}>10 \mathrm{MeV} \\
{\left[\mathrm{cm}^{-2} \mathrm{~s}^{-1} \mathrm{sr}^{-1}\right]}\end{array}$ & $\begin{array}{r}\mathrm{E}>60 \mathrm{MeV} \\
{\left[\mathrm{cm}^{-2} \mathrm{~s}^{-1} \mathrm{sr}^{-1}\right]}\end{array}$ & Magnitude & Location & $\begin{array}{r}\text { Speed } \\
{\left[\mathrm{km} \mathrm{s}^{-1}\right]}\end{array}$ & $\begin{array}{r}\text { Width } \\
\text { [degrees] }\end{array}$ \\
\hline $2003-10-28$ & $12: 00$ & 5600 & 360 & $\mathrm{X} 17$ & $08 \mathrm{E}, 16 \mathrm{~S}$ & 2459 & 360 \\
\hline 2003-10-29 & $22: 00$ & 2700 & 170 & $\mathrm{X} 10$ & $02 \mathrm{~W}, 15 \mathrm{~N}$ & 2029 & 360 \\
\hline 2003-11-02 & $18: 00$ & 1400 & 80 & $\mathrm{X} 8.3$ & $56 \mathrm{~W}, 14 \mathrm{~N}$ & 2598 & 130 \\
\hline 2003-11-04 & 21:00 & 390 & 4.5 & $\mathrm{X} 28$ & $83 \mathrm{~W}, 19 \mathrm{~N}$ & 2657 & 130 \\
\hline 2003-11-20 & $07: 15$ & 17 & 0.16 & M9.6 & $08 \mathrm{~W}, 03 \mathrm{~N}$ & 669 & 90 \\
\hline 2004-07-25 & $16: 00$ & 88 & 0.51 & M2.2 & $33 \mathrm{~W}, 04 \mathrm{~N}$ & 1333 & 130 \\
\hline 2004-09-19 & $18: 05$ & 61 & 1.1 & M1.9 & $58 \mathrm{~W}, 03 \mathrm{~N}$ & - & - \\
\hline 2004-11-07 & $02: 50$ & 6.1 & 0.0050 & M1.9 & $02 \mathrm{~W}, 07 \mathrm{~N}$ & - & - \\
\hline 2004-11-07 & $17: 00$ & 550 & 1.9 & $\mathrm{X} 2.0$ & $17 \mathrm{~W}, 09 \mathrm{~N}$ & 1759 & 150 \\
\hline 2004-11-09 & 19:00 & 89 & 0.53 & M8.9 & $51 \mathrm{~W}, 07 \mathrm{~N}$ & 2000 & 130 \\
\hline 2004-11-10 & 03:00 & 450 & 5.3 & $\mathrm{X} 2.5$ & $49 \mathrm{~W}, 09 \mathrm{~N}$ & 2000 & 120 \\
\hline 2005-01-15 & $08: 25$ & 12 & 0.39 & M8.6 & $06 \mathrm{E}, 11 \mathrm{~S}$ & 2049 & 90 \\
\hline 2005-01-15 & $23: 00$ & 440 & 3.1 & $\mathrm{X} 2.6$ & $05 \mathrm{~W}, 15 \mathrm{~N}$ & 2861 & 130 \\
\hline 2005-01-17 & 10:00 & 4600 & 110 & X3.8 & $25 \mathrm{~W}, 15 \mathrm{~N}$ & 2094 & 110 \\
\hline 2005-01-20 & 06:00 & 2800 & 950 & $\mathrm{X} 7.1$ & $61 \mathrm{~W}, 14 \mathrm{~N}$ & 882 & 80 \\
\hline 2005-05-06 & $12: 00$ & 4.8 & 0.022 & M1.3 & $76 \mathrm{~W}, 04 \mathrm{~N}$ & 1144 & 30 \\
\hline 2005-05-13 & $21: 00$ & 430 & 0.086 & M8.0 & $11 \mathrm{E}, 12 \mathrm{~S}$ & 1689 & 360 \\
\hline 2005-06-16 & $20: 50$ & 47 & 4.2 & M4.0 & $85 \mathrm{~W}, 09 \mathrm{~N}$ & - & - \\
\hline 2005-07-13 & $18: 10$ & 16 & 0.25 & M5.0 & $78 \mathrm{~W}, 11 \mathrm{~N}$ & 1423 & 70 \\
\hline $2005-07-14$ & $11: 00$ & 130 & 1.1 & $\mathrm{X} 1.2$ & $89 \mathrm{~W}, 08 \mathrm{~N}$ & 2115 & 80 \\
\hline $2005-08-22$ & $03: 35$ & 9.4 & 0.19 & M2.6 & $48 \mathrm{~W}, 09 \mathrm{~N}$ & 1194 & 160 \\
\hline 2005-08-22 & $18: 00$ & 320 & 1.8 & M5.6 & $60 \mathrm{~W}, 12 \mathrm{~N}$ & 2378 & 100 \\
\hline
\end{tabular}


Table 4 - The SSE list and associated solar parameters (continued)

\begin{tabular}{|c|c|c|c|c|c|c|c|}
\hline \multicolumn{2}{|c|}{ SEP Onset } & \multicolumn{2}{|c|}{ Proton Peak Flux } & \multicolumn{2}{|c|}{ Flare Parameters } & \multicolumn{2}{|c|}{ CME Parameters } \\
\hline $\begin{array}{c}\text { Date } \\
\text { YYYY-MM-DD }\end{array}$ & $\begin{array}{l}\text { Time } \\
\text { HH:SS }\end{array}$ & $\begin{array}{r}\mathrm{E}>10 \mathrm{MeV} \\
{\left[\mathrm{cm}^{-2} \mathrm{~s}^{-1} \mathrm{sr}^{-1}\right]}\end{array}$ & $\begin{array}{r}\mathrm{E}>60 \mathrm{MeV} \\
{\left[\mathrm{cm}^{-2} \mathrm{~s}^{-1} \mathrm{sr}^{-1}\right]}\end{array}$ & Magnitude & Location & $\begin{array}{r}\text { Speed } \\
{\left[\mathrm{km} \mathrm{s}^{-1}\right]}\end{array}$ & $\begin{array}{r}\text { Width } \\
\text { [degrees] }\end{array}$ \\
\hline 2005-09-07 & $23: 25$ & 1100 & 19 & $\mathrm{X} 17$ & $89 \mathrm{E}, 06 \mathrm{~S}$ & - & - \\
\hline 2005-09-13 & $22: 00$ & 200 & 0.88 & $\mathrm{X} 1.5$ & 10E,09S & 1866 & 130 \\
\hline 2006-12-05 & $17: 35$ & 25 & 0.98 & $\mathrm{X} 9.0$ & $68 \mathrm{E}, 07 \mathrm{~S}$ & - & - \\
\hline 2006-12-06 & $22: 00$ & 2300 & 42 & $\mathrm{X} 6.5$ & $64 \mathrm{E}, 06 \mathrm{~S}$ & - & - \\
\hline 2006-12-13 & 02:00 & 740 & 110 & X 3.4 & $23 \mathrm{~W}, 06 \mathrm{~N}$ & 1774 & 180 \\
\hline 2006-12-14 & $22: 00$ & 230 & 5.4 & $\mathrm{X} 1.5$ & $46 \mathrm{~W}, 07 \mathrm{~N}$ & 1042 & 70 \\
\hline
\end{tabular}


Table 5. Probabilities of SEP occurrence and their respective errors as a function of flare magnitude derived from the CRR2010 list (roman fonts) and the SSE list (italic fonts).

\begin{tabular}{ccccc}
\hline \multirow{5}{*}{ M1-M3.9 } & M4-M6.9 & M7-M9.9 & X1-X4.9 & $\geq$ X5 \\
\hline $0.062 \pm 0.008$ & $0.20 \pm 0.03$ & $0.30 \pm 0.07$ & $0.44 \pm 0.05$ & $0.67 \pm 0.11$ \\
$0.024 \pm 0.005$ & $0.10 \pm 0.03$ & $0.24 \pm 0.06$ & $0.28 \pm 0.05$ & $0.67 \pm 0.11$ \\
\hline
\end{tabular}

Table 6. Probabilities of SEP occurrence and their respective errors as a function of flare magnitude and flare longitude derived from the CRR2010 list (roman fonts) and the SSE list (italic fonts).

\begin{tabular}{cccccc}
\hline Flare longitude & \multicolumn{5}{c}{ Flare magnitude } \\
& M1-M3.9 & M4-M6.9 & M7-M9.9 & X1-X4.9 & $\geq$ X5 \\
\cline { 2 - 6 }$\left[-90^{\circ},-71^{\circ}\right]$ & $0.03 \pm 0.02$ & 0. & 0. & $0.3 \pm 0.19$ & $0.43 \pm 0.27$ \\
& 0. & 0. & 0. & 0. & $0.33 \pm 0.27$ \\
{$\left[-70^{\circ},-31^{\circ}\right]$} & $0.014 \pm 0.008$ & $0.17 \pm 0.07$ & $0.18 \pm 0.12$ & $0.09 \pm 0.06$ & $0.40 \pm 0.22$ \\
& $0.005 \pm 0.005$ & $0.07 \pm 0.05$ & $0.09 \pm 0.09$ & 0. & $0.40 \pm 0.22$ \\
{$\left[-30^{\circ}, 29^{\circ}\right]$} & $0.06 \pm 0.01$ & $0.22 \pm 0.06$ & $0.30 \pm 0.10$ & $0.60 \pm 0.08$ & $0.75 \pm 0.22$ \\
& $0.020 \pm 0.008$ & $0.10 \pm 0.04$ & $0.22 \pm 0.09$ & $0.51 \pm 0.08$ & $0.75 \pm 0.22$ \\
{$\left[30^{\circ}, 69^{\circ}\right]$} & $0.10 \pm 0.02$ & $0.21 \pm 0.07$ & $0.50 \pm 0.16$ & $0.48 \pm 0.10$ & 1. \\
{$\left[70^{\circ}, 90^{\circ}\right]$} & $0.05 \pm 0.01$ & $0.12 \pm 0.06$ & $0.50 \pm 0.16$ & $0.30 \pm 0.10$ & 1. \\
& $0.10 \pm 0.03$ & $0.42 \pm 0.14$ & $0.20 \pm 0.18$ & $0.58 \pm 0.14$ & 1. \\
& $0.032 \pm 0.016$ & $0.25 \pm 0.13$ & $0.20 \pm 0.18$ & $0.25 \pm 0.13$ & 1. \\
\hline
\end{tabular}

Table 7. Probabilities of SEP occurrence and their respective errors as a function of flare magnitude for halo CMEs.

\begin{tabular}{ccc}
\hline \multicolumn{3}{c}{ Flare magnitude } \\
M1-M3.9 & M4-M9.9 & $\geq \mathrm{X} 1$ \\
\hline $0.48 \pm 0.07$ & $0.64 \pm 0.08$ & $0.62 \pm 0.07$ \\
\hline
\end{tabular}

Table 8. Probabilities of SEP occurrence and their respective errors as a function of flare magnitude and CME velocity for non-halo CMEs.

\begin{tabular}{cccc}
\hline & \multicolumn{3}{c}{ CME velocity } \\
& $0-499 \mathrm{~km} \mathrm{~s}^{-1}$ & $500-999 \mathrm{~km} \mathrm{~s}^{-1}$ & $\geq 1000 \mathrm{~km} \mathrm{~s}^{-1}$ \\
\cline { 2 - 4 } M1-M3.9 & $0.03 \pm 0.02$ & $0.10 \pm 0.03$ & $0.39 \pm 0.10$ \\
M4-M9.9 & $0.11 \pm 0.11$ & $0.22 \pm 0.09$ & $0.25 \pm 0.15$ \\
$\geq$ X1 & $0.09 \pm 0.09$ & $0.25 \pm 0.15$ & $0.60 \pm 0.16$ \\
\hline
\end{tabular}


Table 9. Probabilities of SEP occurrence and their respective errors as a function of flare magnitude and longitude for halo CMEs.

\begin{tabular}{cccc}
\hline & \multicolumn{3}{c}{ Flare magnitude } \\
{$\left[-90^{\circ},-1^{\circ}\right]$} & $0.26 \pm 0.09$ & $0.47 \pm 0.12$ & $0.25 \pm 0.11$ \\
{$\left[0^{\circ}, 90^{\circ}\right]$} & $0.67 \pm 0.09$ & $0.82 \pm 0.09$ & $0.79 \pm 0.07$ \\
\hline
\end{tabular}

Table 10. Probabilities of SEP occurrence and their respective errors as a function of flare magnitude, flare longitude and CME velocity for non-halo CMEs.

\begin{tabular}{cccc}
\hline & \multicolumn{3}{c}{ CME velocity } \\
M1-M3.9 and $\left[-90^{\circ},-1^{\circ}\right]$ & $0-499 \mathrm{~km} \mathrm{~s}^{-1}$ & $500-999 \mathrm{~km} \mathrm{~s}^{-1}$ & $\geq 1000 \mathrm{~km} \mathrm{~s}^{-1}$ \\
\cline { 2 - 4 } $\mathrm{M} 4-\mathrm{M} 9.9$ and $\left[-90^{\circ},-1^{\circ}\right]$ & 0 & $0.02 \pm 0.02$ & $0.11 \pm 0.11$ \\
$\geq \mathrm{X} 1$ and $\left[-90^{\circ},-1^{\circ}\right]$ & 0 & $0.13 \pm 0.12$ & $0.25 \pm 0.15$ \\
$\mathrm{M} 1-\mathrm{M} 3.9$ and $\left[0^{\circ}, 90^{\circ}\right]$ & $0.05 \pm 0.30$ & $0.20 \pm 0.18$ & $0.25 \pm 0.22$ \\
M4-M9.9 and $\left[0^{\circ}, 90^{\circ}\right]$ & $0.20 \pm 0.18$ & $0.20 \pm 0.06$ & $0.57 \pm 0.13$ \\
$\geq \mathrm{X} 1$ and $\left[0^{\circ}, 90^{\circ}\right]$ & $0.20 \pm 0.18$ & $0.33 \pm 0.12$ & 0 \\
\hline
\end{tabular}

Table 11. Probabilities of SEP occurrence and their respective errors as a function of CME velocity for all CMEs, non-halo and halo CMEs.

\begin{tabular}{cccc}
\hline & \multicolumn{3}{c}{ CME velocity } \\
& $0-499 \mathrm{~km} \mathrm{~s}^{-1}$ & $500-999 \mathrm{~km} \mathrm{~s}^{-1}$ & $\geq 1000 \mathrm{~km} \mathrm{~s}^{-1}$ \\
\cline { 2 - 4 } all CMEs & $0.06 \pm 0.02$ & $0.22 \pm 0.03$ & $0.56 \pm 0.04$ \\
non-halo CMEs & $0.04 \pm 0.02$ & $0.13 \pm 0.03$ & $0.41 \pm 0.08$ \\
halo CMEs & $0.30 \pm 0.14$ & $0.51 \pm 0.08$ & $0.64 \pm 0.05$ \\
\hline
\end{tabular}

Table 12. Mean and RMS of the logarithm of the proton peak flux for $E>10 \mathrm{MeV}$ and $E>60 \mathrm{MeV}$ in the five flare magnitude bins. Peak fluxes are expressed as $\log \left(\mathrm{cm}^{-2} \mathrm{~s}^{-1} \mathrm{sr}^{-1}\right)$.

\begin{tabular}{ccr}
\hline Flare intensity bin & $\begin{array}{c}\text { Peak flux } \\
E>10 \mathrm{MeV}\end{array}$ & $\begin{array}{c}\text { Peak flux } \\
E>60 \mathrm{MeV}\end{array}$ \\
\hline $\mathrm{M} 1.0 \leq I_{\mathrm{f}}<\mathrm{M} 2.5$ & $1.24 \pm 0.75$ & $-1.38 \pm 1.21$ \\
$\mathrm{M} 2.5 \leq I_{\mathrm{f}}<\mathrm{M} 6.3$ & $1.47 \pm 0.73$ & $-0.49 \pm 0.97$ \\
$\mathrm{M} 6.3 \leq I_{\mathrm{f}}<\mathrm{X} 1.6$ & $1.88 \pm 1.00$ & $-0.07 \pm 1.39$ \\
$\mathrm{X} 1.6 \leq I_{\mathrm{f}}<\mathrm{X} 5.0$ & $2.02 \pm 0.83$ & $0.32 \pm 1.02$ \\
$I_{\mathrm{f}} \geq \mathrm{X} 5.0$ & $3.02 \pm 0.66$ & $1.77 \pm 0.88$ \\
\hline
\end{tabular}


Table 13. Mean and RMS of the logarithm of the proton peak flux for $E>10 \mathrm{MeV}$ and $E>60 \mathrm{MeV}$ in the five CME speed bins. Peak fluxes are expressed as $\log \left(\mathrm{cm}^{-2} \mathrm{~s}^{-1} \mathrm{sr}^{-1}\right)$.

\begin{tabular}{ccr}
\hline CME speed bin & $\begin{array}{c}\text { Peak flux } \\
E>10 \mathrm{MeV}\end{array}$ & $\begin{array}{c}\text { Peak flux } \\
E>60 \mathrm{MeV}\end{array}$ \\
\hline $400 \leq v_{\mathrm{CME}}<1000 \mathrm{~km} \mathrm{~s}^{-1}$ & $1.21 \pm 0.79$ & $-0.69 \pm 1.41$ \\
$1000 \leq v_{\mathrm{CME}}<1400 \mathrm{~km} \mathrm{~s}^{-1}$ & $1.43 \pm 0.61$ & $-0.56 \pm 1.11$ \\
$1400 \leq v_{\mathrm{CME}}<1800 \mathrm{~km} \mathrm{~s}^{-1}$ & $2.21 \pm 1.15$ & $0.22 \pm 1.85$ \\
$1800 \leq v_{\mathrm{CME}}<2200 \mathrm{~km} \mathrm{~s}^{-1}$ & $2.39 \pm 0.79$ & $0.57 \pm 1.17$ \\
$v_{\mathrm{CME}} \geq 2200 \mathrm{~km} \mathrm{~s}^{-1}$ & $2.66 \pm 0.73$ & $0.84 \pm 1.14$ \\
\hline
\end{tabular}

Table 14. Mean and RMS of the logarithm of the proton peak flux in the nine flare magnitude and location bins. Peak fluxes are expressed as $\log \left(\mathrm{cm}^{-2} \mathrm{~s}^{-1} \mathrm{sr}^{-1}\right)$ for $E>10 \mathrm{MeV}$ and $E>60 \mathrm{MeV}$.

\begin{tabular}{rccc}
\hline & $\mathrm{M} 1.0 \leq I_{\mathrm{f}}<\mathrm{M} 3.2$ & $\mathrm{M} 3.2 \leq I_{\mathrm{f}}<\mathrm{X} 1.0$ & $I_{\mathrm{f}} \geq \mathrm{X} 1.0$ \\
\hline \multicolumn{4}{c}{ proton peak flux $E>10 \mathrm{MeV}$} \\
$-90^{\circ} \leq L_{\mathrm{f}}<-30^{\circ}$ & $1.46 \pm 1.46$ & $1.09 \pm 0.58$ & $2.60 \pm 1.06$ \\
$-30^{\circ} \leq L_{\mathrm{f}}<30^{\circ}$ & $0.99 \pm 0.65$ & $1.46 \pm 0.88$ & $2.27 \pm 1.01$ \\
$30^{\circ} \leq L_{\mathrm{f}}<90^{\circ}$ & $1.51 \pm 0.84$ & $1.82 \pm 0.99$ & $2.46 \pm 0.73$ \\
\hline \multicolumn{4}{c}{ proton peak flux $E>60 \mathrm{MeV}$} \\
$-90^{\circ} \leq L_{\mathrm{f}}<-30^{\circ}$ & $-1.80 \pm 1.80$ & $-1.25 \pm 0.24$ & $0.97 \pm 0.86$ \\
$-30^{\circ} \leq L_{\mathrm{f}}<30^{\circ}$ & $-1.79 \pm 0.80$ & $-0.68 \pm 1.22$ & $0.56 \pm 1.31$ \\
$30^{\circ} \leq L_{\mathrm{f}}<90^{\circ}$ & $-0.86 \pm 1.34$ & $0.13 \pm 1.35$ & $1.05 \pm 0.98$ \\
\hline
\end{tabular}

Table 15. Mean and RMS of the logarithm of the proton peak flux in the nine flare magnitude and CME speed bins. Peak fluxes are expressed as $\log \left(\mathrm{cm}^{-2} \mathrm{~s}^{-1} \mathrm{sr}^{-1}\right)$ for $E>10 \mathrm{MeV}$ and $E>60 \mathrm{MeV}$.

\begin{tabular}{cccc}
\hline & $\mathrm{M} 1.0 \leq I_{\mathrm{f}}<\mathrm{M} 3.2$ & $\mathrm{M} 3.2 \leq I_{\mathrm{f}}<\mathrm{X} 1.0$ & $I_{\mathrm{f}} \geq \mathrm{X} 1.0$ \\
\hline \multicolumn{4}{c}{ proton peak flux $E>10 \mathrm{MeV}$} \\
$400 \leq v_{\mathrm{CME}}<1200 \mathrm{kms}^{-1}$ & $0.93 \pm 0.40$ & $0.96 \pm 0.48$ & $1.81 \pm 0.83$ \\
$1200 \leq v_{\mathrm{CME}}<2000 \mathrm{kms}^{-1}$ & $1.67 \pm 0.76$ & $1.87 \pm 1.10$ & $2.34 \pm 0.85$ \\
$v_{\mathrm{CME}} \geq 2000 \mathrm{kms}^{-1}$ & - & $1.72 \pm 0.55$ & $2.97 \pm 0.56$ \\
\hline & \multicolumn{4}{c}{ proton peak flux $E>60 \mathrm{MeV}$} \\
$400 \leq v_{\mathrm{CME}}<1200 \mathrm{kms}^{-1}$ & $-1.47 \pm 0.64$ & $-1.12 \pm 1.14$ & $0.31 \pm 1.30$ \\
$1200 \leq v_{\mathrm{CME}}<2000 \mathrm{kms}^{-1}$ & $-1.13 \pm 1.47$ & $-0.03 \pm 1.48$ & $0.79 \pm 1.18$ \\
$v_{\mathrm{CME}} \geq 2000 \mathrm{kms}^{-1}$ & - & $-0.39 \pm 0.50$ & $1.30 \pm 0.89$ \\
\hline
\end{tabular}


Table 16. Mean and RMS of the logarithm of the proton peak flux in the nine flare magnitude and CME width bins. Peak fluxes are expressed as $\log \left(\mathrm{cm}^{-2} \mathrm{~s}^{-1} \mathrm{sr}^{-1}\right)$ for $E>10 \mathrm{MeV}$ and $E>60 \mathrm{MeV}$.

\begin{tabular}{cccc}
\hline & $\mathrm{M} 1.0 \leq I_{\mathrm{f}}<\mathrm{M} 3.2$ & $\mathrm{M} 3.2 \leq I_{\mathrm{f}}<\mathrm{X} 1.0$ & $I_{\mathrm{f}} \geq \mathrm{X} 1.0$ \\
\hline \multicolumn{4}{c}{ proton peak flux $E>10 \mathrm{MeV}$} \\
$0^{\circ} \leq w_{\mathrm{CME}}<100^{\circ}$ & $0.92 \pm 0.42$ & $1.41 \pm 0.72$ & $2.13 \pm 1.05$ \\
$100^{\circ} \leq w_{\mathrm{CME}}<360^{\circ}$ & $1.57 \pm 0.80$ & $1.70 \pm 1.13$ & $2.36 \pm 0.79$ \\
$w_{\mathrm{CME}}=360^{\circ}$ & $1.18 \pm 1.18$ & $1.68 \pm 1.09$ & $2.49 \pm 1.16$ \\
\hline \multicolumn{4}{c}{ proton peak flux $E>60 \mathrm{MeV}$} \\
$0^{\circ} \leq w_{\mathrm{CME}}<100^{\circ}$ & $-1.79 \pm 0.59$ & $-0.62 \pm 1.15$ & $0.71 \pm 1.51$ \\
$100^{\circ} \leq w_{\mathrm{CME}}<360^{\circ}$ & $-0.95 \pm 1.33$ & $-0.13 \pm 1.44$ & $0.74 \pm 1.07$ \\
$w_{\mathrm{CME}}=360^{\circ}$ & $-1.81 \pm 1.81$ & $-0.58 \pm 1.55$ & $0.99 \pm 1.47$ \\
\hline
\end{tabular}

Table 17. Mean and RMS of the logarithm of the proton peak flux in the six CME speed and width bins. Peak fluxes are expressed as $\log \left(\mathrm{cm}^{-2} \mathrm{~s}^{-1} \mathrm{sr}^{-1}\right)$ for $E>10 \mathrm{MeV}$ and $E>60 \mathrm{MeV}$.

\begin{tabular}{ccc}
\hline & $v_{\mathrm{CME}}<1500 \mathrm{~km} \mathrm{~s}^{-1}$ & $v_{\mathrm{CME}} \geq 1500 \mathrm{~km} \mathrm{~s}^{-1}$ \\
\hline & proton peak flux $E>10 \mathrm{MeV}$ \\
$0^{\circ} \leq w_{\mathrm{CME}}<100^{\circ}$ & $1.42 \pm 0.88$ & $1.61 \pm 0.75$ \\
$100^{\circ} \leq w_{\mathrm{CME}}<360^{\circ}$ & $1.51 \pm 0.76$ & $2.45 \pm 0.88$ \\
$w_{\mathrm{CME}}=360^{\circ}$ & $1.14 \pm 0.49$ & $2.87 \pm 0.89$ \\
\hline \multicolumn{3}{c}{ proton peak flux $E>60 \mathrm{MeV}$} \\
$0^{\circ} \leq w_{\mathrm{CME}}<100^{\circ}$ & $-0.54 \pm 1.52$ & $-0.71 \pm 1.02$ \\
$100^{\circ} \leq w_{\mathrm{CME}}<360^{\circ}$ & $-0.34 \pm 1.19$ & $0.57 \pm 1.41$ \\
$w_{\mathrm{CME}}=360^{\circ}$ & $-0.79 \pm 0.89$ & $0.99 \pm 1.82$ \\
\hline
\end{tabular}




\section{References}

Belov, A., Garcia, H., Kurt, V., Mavromichalaki, H., Gerontidou, M.: 2005, Proton Enhancements and Their Relation to the X-Ray Flares During the Three Last Solar Cycles. Solar Phys. 229, 135-159. doi 10.1007/s11207-005-4721-3

Cane, H.V.: 1995, The structure and evolution of interplanetary shocks and the relevance for particle acceleration. Nuclear Phys. B Proc. Suppl. 39, 35-44. doi $10.1016 / 0920-5632(95) 00005-T$

Cane, H.V., McGuire, R.E., von Rosenvinge, T.T.: 1986, Two classes of solar energetic particle events associated with impulsive and long-duration soft X-ray flares. Astrophys. J. 301, 448 - 459. doi $10.1086 / 163913$

Cane, H.V., Reames, D.V., von Rosenvinge, T.T.: 1988, The role of interplanetary shocks in the longitude distribution of solar energetic particles. J. Geophys. Res. 93, 9555-9567. doi $10.1029 /$ JA093iA09p09555.

Cane, H.V., Richardson, I.G., von Rosenvinge, T.T.: 2010, A study of solar energetic particle events of 1997-2006: Their composition and associations. J. Geophys. Res. (Space Physics) 115, 8101. doi 10.1029/2009JA014848

Channok, C., Ruffolo, D., Desai, M.I., Mason, G.M.: 2005, Finite-Time Shock Acceleration of Energetic Storm Particles. Astrophys. J. Lett. 633, L53-L56. doi 10.1086/498108

Cliver, E.W., Ling, A.G., Belov, A., Yashiro, S.: 2012, Size distributions of solar flares and solar energetic particle events. Astrophys. J. Lett. 756, L29. doi 10.1088/2041-8205/756/2/L29.

Crosby, N.B., Veronig, A., Robbrecht, E., Vrsnak, B., Vennerstrom, S., Malandraki, O., Dalla, S., Rodriguez, L., Srivastava, N., Hesse, M., Odstrcil, D., COMESEP Consortium: 2012, Forecasting the space weather impact: The COMESEP project. In: Hu, Q., Li, G., Zank, G.P., Ao, X., Verkhoglyadova, O., Adams, J.H. (eds.) Amer. Inst. Physics. C. S. 1500, 159 - 164 . doi $10.1063 / 1.4768760$

Crosby, N.B., Heynderickx, D., Jiggens, P., Aran. A., B. Sanahuja, Truscott, P., Lei, F., Jacobs, J., Poedts, S., Gabriel, S., Sandberg, I., Glover, A., Hilgers, A.: 2014, SEPEM: a tool for statistical modelling the solar energetic particle environment. Space Weather, submitted.

Dalla, S., Agueda, N.: 2010, Role of latitude of source region in Solar Energetic Particle events. Twelfth Int. Solar Wind Conf. 1216, 613-616. doi 10.1063/1.3395941

Dumbovic, M., Devos, A., Vrsnak, B., Sudar, D., Rodriguez, L., Ruzdjak, D., Leer, K., Vennestrom, S., Veronig, A.M., Robbrecht, E.: 2014, Geoeffectiveness of Coronal Mass Ejections in the SOHO era. Solar Phys., accepted.

Gopalswamy, N., Yashiro, S., Akiyama, S., Mäkelä, P., Xie, H., Kaiser, M.L., Howard, R.A., Bougeret, J.L.: 2008, Coronal mass ejections, type II radio bursts, and solar energetic particle events in the SOHO era. Annales Geophys. 26, 3033-3047. doi 10.5194/angeo-26-3033-2008

Hwang, J., Cho, K.-S., Moon, Y.-J., Kim, R.-S., Park, Y.-D.: 2010, Solar proton events during the solar cycle 23 and their association with CME parameters. Acta Astron. 67, 353-361.

Jiggens, P.T.A., Gabriel, S.B., Heynderickx, D., Crosby, N., Glover, A., Hilgers, A.: 2012, ESA SEPEM Project: Peak Flux and Fluence Model. IEEE Transac. Nuclear Sc. 59, 1066-1077. doi: 10.1109 /TNS.2012.2198242

Kahler, S.W.: 2001, The correlation between solar energetic particle peak intensities and speeds of coronal mass ejections: Effects of ambient particle intensities and energy spectra. J. Geophys. Res. 106, 20947-20956. doi 10.1029/2000JA002231

Kallenrode, M.-B.: 2003, Current views on impulsive and gradual solar energetic particle events. J. Phys. G, Nuclear Phys. 29, 965-981.

Kocharov, L., Laitinen, T., Usoskin, I., Vainio, R.: 2014, Transmission and Emission of Solar Energetic Particles in Semi-transparent Shocks. Astrophys. J. Lett. 787, L21. doi $10.1088 / 2041-8205 / 787 / 2 /$ L21

Kurt, V., Belov, A., Mavromichalaki, H., Gerontidou, M.: 2004, Statistical analysis of solar proton events. Annales Geophys. 22, 2255 -2271. doi 10.5194/angeo-22-2255-2004

Laurenza, M., Cliver, E.W., Hewitt, J., Storini, M., Ling, A.G., Balch, C.C., Kaiser, M.L.: 2009, A technique for short-term warning of solar energetic particle events based on flare location, flare size, and evidence of particle escape. Space Weather 7, 4008. doi: $10.1029 / 2007$ SW000379

Miteva, R., Klein, K.-L., Malandraki, O., Dorrian, G.: 2013, Solar Energetic Particle Events in the 23rd Solar Cycle: Interplanetary Magnetic Field Configuration and Statistical Relationship with Flares and CMEs. Solar Phys. 282, 579-613. doi $10.1007 /$ s11207-012-0195-2 
Park, J., Moon, Y.-J., Gopalswamy, N.: 2012, Dependence of solar proton events on their associated activities: Coronal mass ejection parameters. J. Geophys. Res. (Space Physics) 117, 8108. doi 10.1029/2011JA017477

Park, J., Moon, Y.-J., Lee, D.H., Youn, S.: 2010, Dependence of solar proton events on their associated activities: Flare parameters. J. Geophys. Res. (Space Physics) 115(A14), 10105. doi $10.1029 / 2010$ JA015330.

Reames, D.V.: 1988, Bimodal abundances in the energetic particles of solar and interplanetary origin. Astrophys. J. Lett. 330, L71-L75. doi $10.1086 / 185207$

Reames, D.V.: 1999, Particle acceleration at the Sun and in the heliosphere. Space Sci. Rev. 90, 413-491. doi 10.1023/A:1005105831781

Reames, D.V.: 2013, The Two Sources of Solar Energetic Particles. Space Sci. Rev. 175, 53 - 92. doi $10.1007 /$ s11214-013-9958-9

Richardson, I.G., von Rosenvinge, T.T., Cane, H.V., Christian, E.R., Cohen, C.M.S., Labrador, A.W., Leske, R.A., Mewaldt, R.A., Wiedenbeck, M.E., Stone, E.C.: 2014, > 25 MeV proton events observed by the High Energy Telescopes on the STEREO A and B spacecraft and/or at Earth during the first $\sim$ seven years of the STEREO mission. Solar Phys.. doi $10.1007 /$ s11207-014-0524-8

Trottet, G., Samwel, S., Klein, K.-L.., Dudok de Wit, T., Miteva, R.: 2014, Statistical evidence for contributions of flares and coronal mass ejections to major solar energetic particle events. Solar Phys., accepted.

Van Hollebeke, M.A.I., Ma Sung, L.S., McDonald, F.B.: 1975, The variation of solar proton energy spectra and size distribution with heliolongitude. Solar Phys. 41, 189-223. doi:10.1007/BF00152967

Wall, J.V., Jenkins, C.R.: 2003, Practical Statistics for Astronomers, Cambridge University Press, Cambridge.

Wang, R.: 2006, Statistical characteristics of solar energetic proton events from January 1997 to June 2005. Astroparticle Phys. 26, 202 - 208. doi 10.1016/j.astropartphys.2006.06.003

Xapsos, M.A., Barth, J.L., Stassinopoulos, E.G., Messenger, S.R., Walters, R.J., Summers, G.P., Burke, E.A.: 2000, Characterizing solar proton energy spectra for radiation effects applications. IEEE Transac. Nuclear Sc. 47, 2218-2223. doi 10.1109/23.903756 
SOLA: sep_analysis.tex; 2 October 2018; 9:47; p. 40 